\title{
Comparison of the TESLA, NLC and CLIC Beam Collimation System Performance
}

\begin{abstract}
A. Drozhdin ${ }^{4}$, G. Blair ${ }^{6}$, L. Keller ${ }^{5}$, W. Kozanecki ${ }^{2}$, T. Markiewicz ${ }^{5}$, T. Murayama $^{5}$, N. Mokhov ${ }^{4}$, O. Napoly ${ }^{2}$, T. Raubenheimer ${ }^{5}$, D. Schulte ${ }^{3}$, A. Seryi ${ }^{5}$, P. Tenenbaum ${ }^{5}$, N. Walker ${ }^{1}$, M. Woodley ${ }^{5}$, F. Zimmermann ${ }^{3}$
\end{abstract}

1. DESY, Hamburg, Germany

2. CEA-Saclay, Paris, France

3. CERN, Geneva, Switzerland

4. Fermilab, Batavia, Illinois, USA

5. SLAC, Menlo Park, CA, USA

6. University of London, London, United Kingdom

\section{March 2003}

Abstract: This note describes studies performed in the framework of the Collimation Task Force organized to support the work of the International Linear Collider Technical Review Committee. The post-linac beam-collimation systems in the TESLA, JLC/NLC and CLIC linear-collider designs are compared using the same computer code under the same assumptions. Their performance is quantified in terms of beam-halo and synchrotron-radiation collimation efficiency. The performance of the current designs varies across projects and does not always meet the original design goals. However, these comparisons suggest that achieving the required performance in a future linear collider is feasible. The post-TRC plans of the Collimation Task Force are outlined briefly in closing. 


\title{
Comparison of the TESLA, NLC and CLIC Beam-Collimation System Performance
}

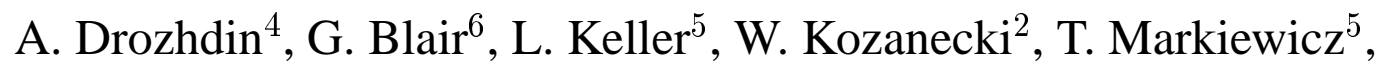

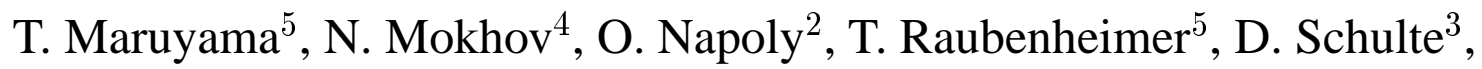

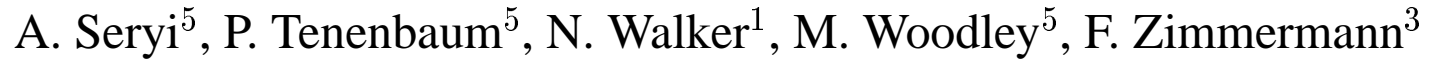 \\ ${ }^{1}$ DESY, Germany; ${ }^{2}$ CEA-Saclay, France; ${ }^{3}$ CERN, Switzerland; \\ ${ }^{4}$ FNAL, USA; ${ }^{5}$ SLAC, USA; ${ }^{6}$ Univ. of London, Great Britain
}

\author{
TESLA Report 2003-02, DESY \\ CLIC Note-555, CERN \\ CERN-AB-Report-2003-006 \\ FERMILAB-TM-2200, FNAL \\ LCC-Note-0111, SLAC
}

March 7, 2003

\begin{abstract}
This note describes studies performed in the framework of the Collimation Task Force organized to support the work of the International Linear Collider Technical Review Committee. The post-linac beam-collimation systems in the TESLA, JLC/NLC and CLIC linear-collider designs are compared using the same computer code under the same assumptions. Their performance is quantified in terms of beam-halo and synchrotron-radiation collimation efficiency. The performance of the current designs varies across projects, and does not always meet the original design goals. But these comparisons suggest that achieving the required performance in a future linear collider is feasible. The post-TRC plans of the Collimation Task Force are briefly outlined in closing.
\end{abstract}

\section{Introduction}

At the nominal parameters of the next generation $e^{+} e^{-}$linear colliders (see Ref. [1] and Table 1), small fractional beam losses along the transport line, or the presence of particles far from the beam core in the IP region, may strongly affect the background conditions in the detector, as well as cause irradiation and heating of collider components. The beam halo, which may extend many standard deviations beyond the beam core, can result in electromagnetic showers and synchrotron 
radiation reaching the detector, as well as in the generation of muon background from particles that are intercepted by physical apertures along the entire beam line.

All machine designs need to remove this halo to a certain "collimation depth", which is generally set by the synchrotron-radiation fan generated by the halo particles in the last few magnets close to the IP: by definition, all particles within the collimation depth generate photons that should pass cleanly through the IR. Halo particles outside of the required collimation depth are removed by physically intercepting them with "collimators", which are formed by a thick absorber of many radiation lengths placed in the optical shadow of a thin spoiler, the thickness of which is generally less than one radiation length. In principle, the loss of particles from the halo should be at controlled points along the lattice, i.e. at the absorbers. These locations then become sources of muons. How many of these muons eventually reach the detector depends on many factors: distance from source to the IP, beam-line layout, tunnel geometry and the use of "muon spoilers".

We present here a comparison of the collimation-system performance for the three main candidates of this generation: the JLC/NLC and CLIC designs based on high-frequency room-temperature rf accelerators and the TESLA design based on a low-frequency superconducting rf accelerator. The loss pattern from the beam halo and the synchrotron-radiation loads (both from the halo and from the core of the beam) are calculated along the beamline and in the IP region. The actual background conditions in the detector (e.g. the number of muons reaching the IR hall) are not evaluated in this paper.

One of the biggest uncertainties affecting such comparisons is the absolute intensity and the phasespace distribution of the beam halo that one has to assume. The $10^{-3}$ fraction of the beam observed in the halo at the SLC (when it was working well) was never quantitatively understood, although it might be explainable by the absence of pre-linac collimation and by tails coming from the damping rings. Analytic estimates performed for high-energy linear colliders predict a much smaller halo, of the order of $10^{-6}$ of the beam current. However, given the SLC experience, designers of collimation systems have taken the conservative approach to build a collimation system that would be able to intercept a fractional halo of $10^{-3}$ of the beam. In terms of average power, fully scraping such a halo would dissipate $11.3 \mathrm{~kW}$ for TESLA, $6.9 \mathrm{~kW}$ for NLC and $4.9 \mathrm{~kW}$ for CLIC (for each beam).

\section{Description of the Collimation Systems}

The functional requirements of the collimation system can be summarized as follows.

1. Losses of halo particles in the final focus system must be limited to a level that results in a tolerable muon flux in the detector.

2. The beam halo in the final doublet (FD) must be limited to a certain maximum transverse size, such that all the synchrotron radiation (SR) passes freely through the IP region and cleanly exits the nearby magnets of the outgoing-beam line (i.e. the first few magnets of the spent-beam extraction line for crossing-angle machines, or the opposite-side final doublet in the case of a head-on geometry).

3. The beamline must be protected against damage by as little as one off-energy beam bunch coming from the linac. 
It is not possible to stop all the halo particles: edge scattering, non-linear fields at high transverse amplitudes, etc. tend to repopulate the phase space outside the collimation depth. All machines currently have a dedicated primary collimation system located upstream of the final focus system (FFS). Additional secondary or "clean-up" collimators are located in the FFS. The maximum number of halo particles that may be intercepted in this secondary system is limited by the muon flux the detector can tolerate. The primary system-which intercepts most of the halo - should have high enough an "efficiency" to reduce the losses in the secondary system to acceptable levels. At the same time, the combination of primary and secondary collimation must bring the halo population outside the collimation depth in the final doublets within tolerance. It is typically required that no SR photon (whether produced by the beam core or by the halo) be allowed to hit any detector or machine component between the entrance to the final doublet on the incoming-beam side, and the exit of the opposite-side final doublet (or of the equivalent magnetic elements in the spent-beam line in the case of a crossing-angle geometry). In addition, no charged halo particles should be allowed to hit material in the same region.

Collimation of the beam requires putting material very close to a beam with a very high energy density, which in turn creates a risk that a missteered beam might destroy the collimator. In principle, the collimators can be protected from damage by enlarging the transverse dimensions of the beam at the collimator locations. In practice, in order to limit the betatron functions in the collimation region, the design relies on thin (0.5-1 radiation length) spoilers which scrape the halo with minimal heating and enlarge the spot size of a missteered beam via multiple Coulomb scattering and energy loss. The enlarged beam is then absorbed in thick (30 radiation lengths) copper absorbers. Absorbers in the primary collimation section should lie in the shadow of their spoiler partner; their aperture should be as large as possible to reduce the probability of being hit directly by a missteered beam, while remaining tight enough to intercept those halo particles that scattered in the spoilers.

An additional concern is that spoilers and absorbers close to the beam may introduce transverse wakefield deflections [2], which would unacceptably degrade the beam quality at the IP. The design of the NLC spoiler is a rotating wheel made of copper and beryllium (Figure 1). The wheel contains a 0.5 radiation length slab of copper in the longitudinal center, with tapered pieces of $\mathrm{Cu}-$ coated beryllium on either side. From the collimation point of view, the spoiler looks like a thin scatterer, while from the wakefield viewpoint, it is a long, tapered metal object which minimizes the wakefield kick.

Parameters of the collimation systems in TESLA, NLC, and CLIC are listed in Tables 2, 3, and 4 respectively. Table 5 lists the physical properties of the spoilers and absorbers for the three machines. Figures 2, 3 and 4 show the collimator locations, the horizontal dispersion, and the collimator apertures. The IR-aperture models used for simulating the TESLA and NLC IRs are shown in Figures 5 and 6 . The SR requirements determine the collimation apertures in both planes at high- $\beta$ points in the collimation system. This leads to an effective collimation depth of the "sine-like" trajectories (with respect to the IP) at spoiler settings of $13 \sigma_{x}$ and $80 \sigma_{y}$ for TESLA, $15 \sigma_{x}$ and $31 \sigma_{y}$ for NLC, and $11 \sigma_{x}$ and $100 \sigma_{y}$ for CLIC. In some cases the spoiler settings must be tighter than the effective collimation depth because of dispersive or higher-order effects. The need for off-energy collimation leads to the requirement of a high-dispersion point in the system, where an off-energy pulse can be safely absorbed.

In TESLA the halo is collimated by the betatron collimation system at every 45 degrees in both planes. The system consists of four (35 mm long) "frame shape" titanium spoilers and four $(0.5$ 
m long) copper absorbers $[3,4,5]$. The spoilers are located at approximately $12 \sigma_{x}$ and $74 \sigma_{y}$ with a safety factor of $1 / \cos (\pi / 8)$ in a region with large horizontal and vertical $\beta$-functions. Energy collimation is located upstream of the betatron collimation section. It consists of a set of nonlinear magnets (sextupoles and octupole) and an off-energy spoiler "spo-m2"and absorber "abs-m2" placed at $1.5 \%$ energy deviation in a region with maximum dispersion (Figure 3). Apertures of the beam delivery section used in the simulations are shown in Figures 4 and 5. It should be noted that the aperture limitation associated with the TESLA vertex detector (whose nominal radius is $15 \mathrm{~mm}$ ) was not included into the geometry model (the smallest apertures near the IP are the detector masks with $12 \mathrm{~mm}$ radius).

In NLC the betatron collimation system consists of five spoiler-absorber sets. Energy collimation is done in a high dispersion region downstream of the betatron collimation system [6]. The spoiler gaps are set to $10 \sigma_{x}$ and $31 \sigma_{y}$. A recent development in the NLC collimation system is the use of octupole doublets which permit the beam halo in one betatron phase to be reduced in amplitude, while leaving the beam core nearly unaffected. A pair of these doublets has been shown to reduce the transverse size of the halo, at the critical final doublet betatron phase, by a factor of four. Simulations performed with these octupoles $\mathrm{ON}$ yielded encouraging results. However, in order to limit our comparisons to a single configuration per linear collider concept, only the (more pessimistic) case of NLC collimation with octupoles OFF is presented in this paper.

In CLIC the collimation system consists of an upstream energy collimation system, based on a dogleg bend, followed by a betatron collimation system [7]. The spoiler half-gaps are set at $9 \sigma_{x}$ and $65 \sigma_{y}$. One should note that CLIC simulations started at a later stage of this comparative study, and that the corresponding system optimization has not been fully completed yet. In particular, the current setting of the energy spoiler $(\mathrm{dP} / \mathrm{P}=0.005)$ is very tight, and results in several percent of the primary beam being intercepted at that location. Although clearly unacceptable in a realistic design, this problem was temporarily neglected, in recognition of the fact that the CLIC-500 optimization is still ongoing.

In all collimation systems a small fraction of the halo escapes from the betatron collimation section and continues into the FFS. This leads to the requirement for a second stage of collimation at high$\beta$ points in the FFS. The secondary-collimation section:

- provides additional safety in suppressing background from large amplitude particles which may escape from the first stage, or may be produced by beam-gas scattering between the collimation section and the FFS;

- keeps the detector background at an acceptable level even for noticeable transverse displacements of the beam centroid in the primary-collimation section;

- cleans the beam of large amplitude "sine-like" trajectories independently of the phase advance between the primary-collimation section and the IP. This leaves open the possibility to modify, at a later stage, upstream portions of the beam delivery section without excessively impacting the collimation efficiency.

\section{Simulation Tools}

Particle tracking and beam loss simulations were carried out using the Program STRUCT [8]. This package performs particle tracking and interaction with collimators in circular accelerators and 
beam lines. Synchrotron radiation and transport along the accelerator are simulated for electron machines. All lattice components with their strength and aperture restrictions are taken into account. This Monte Carlo code, written in Fortran, allows performance analysis of designed lattices, simulation of the beam loss distribution along the accelerator, and other tracking studies. STRUCT has been used for simulations of the Tevatron, Booster and Main Injector at FNAL, for the Japanese JHF project, the LHC at CERN, the SSC, and the collimation system in the Muon Collider.

The accelerator or beam line to be studied is described as a sequence of beam elements placed sequentially along a reference orbit. Every element can be misaligned with respect to the reference system. This misalignment is described by horizontal and vertical displacements, and by rotations around the reference orbit and around the local horizontal and vertical axes.

Physical beam-line elements can include: straight section, quadrupole, rectangular and sector bending magnet, sextupole, octupole, dipole magnet with gradient, electrostatic deflector or septummagnet, RF acceleration, bent crystal, target, collimator, and magnetized collimator.

An aperture definition is required for every lattice element. STRUCT allows five types of aperture for all elements: uniform along the element with rectangular, circular, elliptical and trapezoidal (or pole rotated) cross section. The element may have a variable aperture along its length (a socalled "conical" aperture). In this case the element has a rectangular aperture at any cross section along the element length, and the horizontal and vertical sizes of the aperture are linear functions of length.

As a check, the performance of the NLC collimation system was evaluated with TURTLE [9] and GEANT3 [10]. The agreement with the results from STRUCT was fully satisfactory.

\section{Results}

\subsection{Methodology}

The effectiveness of the collimation system can be quantified in terms of either:

- the fraction of initial halo particles that survive (or are rescattered out of) the primary collimation system and hit secondary collimators or other aperture limitations closer to the IP. This "primary-collimation efficiency" is relevant when estimating muon backgrounds, and is discussed in Sec. 4.2 below;

or

- the number of halo particles that lie outside the collimation depth when they reach the final doublet. This parameter is relevant when estimating synchrotron-radiation backgrounds (as well as the rate of lost-particle hits close to the detector, if any); these are treated in Secs. 4.3 and 4.4 .

For simulations of the effectiveness of the three collimation systems and of background conditions at the IP, the beam halo was represented by a large number of rays (typically $5 \times 10^{5}$ ) distributed in 
phase space in the shape of a ring, with $1 / x$ and $1 / y$ density distributions and covering a machinedependent range of amplitudes $A_{x}$ and $A_{y}$. The range of amplitudes was chosen so as to appropriately overlap the design collimation depth ${ }^{1}$. Such a halo distribution maximizes the fraction of particles that may interact with the spoilers, and it is a more pessimistic assumption than, for example, a uniformly distributed halo with the same maximal range. The halo was also given a Gaussian momentum distribution with typically $\sigma(d P / P)=1 \%$. Parameters of the initial halo distribution are regrouped in Table 6, and examples of the initial distributions are shown in Figs. 7 , 8 and 9.

The main results, summarized in Tables 7-10 and Figures 10-13, are discussed in detail below.

\subsection{Primary-collimation Efficiency}

Figure 10 (left) displays, for each machine, the cumulative particle loss, starting at the IP and integrating back to the entrance of the collimation system.

- The NLC design achieves a primary-collimation efficiency significantly better than $10^{-5}$, resulting in less than $10^{4}$ particles per train ${ }^{2}$ being lost in the secondary system.

- In TESLA, with the primary collimation as currently designed, the loss rate in the secondary system amounts to about $1 \%$ of the initial halo population. Because the TESLA bunch spacing is longer than the entire bunch train for the warm machines, TESLA generally quotes background rates per bunch crossing. However the subdetector most sensitive to muon background, the time projection chamber (TPC), integrates over 150 bunches, so that for the same assumed incident halo fraction of $10^{-3}$, the effective halo population becomes similar to that of NLC and the effective loss in the secondary collimation system amounts to $3 \cdot 10^{7}$ particles per sensitivity window.

- The CLIC collimation system achieves a primary-collimation efficiency of about $3 \times 10^{-4}$.

It should be noted that the primary-collimation efficiency, as defined above, is probably too crude a figure of merit for either NLC or CLIC. In these two designs, the losses are rapidly decreasing near the end of the primary-collimation section, and (within the assumptions of the present simulations) entirely disappear a few ten meters downstream of it. This indicates that the collimation is actually more effective than suggested by the raw numbers in Table 7 . The muon flux reaching the detector (updated simulations of which remain to be carried out) will provide a more relevant measure of primary-collimation performance.

\subsection{Halo Photons}

The collimation-system performance achieved at the entrance to the final doublet, and the resulting level of halo-induced SR backgrounds, are summarized in Figures 10 (right)-12 and Tables 8-9. They can be characterized as follows.

\footnotetext{
${ }^{1}$ It was explicitly checked that the chosen range is large enough to fully populate all accessible regions of phase space. This is illustrated in the Appendix (Figures 31 and 36).

${ }^{2}$ It is unlikely that the simulations are fully accurate down to such low loss levels.
} 
- In NLC, the edge of the collimation depth is sharply defined; but for no halo photons to hit the beam pipe near the IP, rather tight collimator settings $( \pm 0.2-0.3 \mathrm{~mm})$ are needed (in the absence of tail-folding octupoles only).

The halo photon flux hitting the FD SR mask (DUMP2) on the incoming-side (Table 8) is low enough to be of no concern; in addition, these photons are rather soft ( $<E_{\gamma}>\sim 31 \mathrm{KeV}$ ), as illustrated in Figure 11. The halo hitting the detector masks and the vertex detector is negligible (Table 9). Photon losses in the outgoing beam line were not calculated for NLC or CLIC because it was assumed that the crossing-angle geometry provides enough flexibility for an ample stay-clear on the spent-beam side. This assumption needs to be validated by a more complete modelling of the warm-machine IRs.

- In TESLA, the boundary of the collimated halo is barely visible (Figure 10 right), in spite of several tight collimator settings, in particular in the secondary-collimation system (see Table 2). Charged-halo losses on the SR mask DUMP1 amount to about 7400 particles/bunch on the upstream side, and about 250 particles/bunch on the downstream side. In addition, a large number of halo particles $\left(\sim 10^{5} / \mathrm{bunch}\right)$ enter the final doublet outside the collimation depth. ${ }^{3}$ The simulations also indicate that with the collimator configuration simulated here (which corresponds to that of Ref. [11]), some SR photons from the halo $\left(>10^{5}\right.$ photons/bunch) hit the detector mask located $3 \mathrm{~m}$ from the IP; their total energy $(158 \mathrm{GeV} / \mathrm{bunch}$, see Table 9) is however small compared to that of beam-beam induced pairs.

More importantly, one observes a sizeable outgoing photon halo $\left(\sim 1.2 \times 10^{5} \mathrm{GeV} / \mathrm{bunch}\right.$, corresponding to about $1.2 \times 10^{7}$ photons) hitting the downstream SR mask $18 \mathrm{~m}$ from the IP: the total energy of the halo photons intercepted by this mask is about half of that deposited by outgoing SR photons from the beam core hitting the same mask (Table 10). Both the mean energy (Table 8) and the number of halo photons per pulse is an order of magnitude larger in TESLA than in NLC, because of significantly stronger bending fields. This remark also applies to SR photons radiated by the core of the incoming $e^{ \pm}$beam.

It should be noted that the aperture limitation associated with the TESLA vertex detector (whose radius is larger than the radius of the detector masks) was not included in the geometry model and, therefore, part of the flux currently intercepted by the IP beam pipe and the downstream detector mask (see Table 9 and Figure 21 in the Appendix) would actually be intercepted by the vertex detector.

- The halo in CLIC-500 appears reasonably well-behaved, and the number of photons hitting the SR and IR masks is of no concern. This promising performance was however obtained with rather tight collimator settings. But detailed simulations of the $500 \mathrm{GeV}$ CLIC system are only beginning, and its collimator configuration is still very much in flux.

Figure 12 displays the energy spectrum of all halo-induced SR photons at the IP. Although the TESLA spectrum remains the hardest, the mean photon energies in the various LC designs lie within a factor of three of each other. These photons are substantially harder than those hitting the upstream SR mask (Figure 11), underscoring the importance to avoid intercepting them anywhere close to the IP.

\footnotetext{
${ }^{3}$ The apparent contradiction with Figure II.7.5.3 of Ref. [11], in which no particles are found outside the collimation depth, remains to be resolved.
} 


\subsection{Synchrotron Radiation from the Beam Core}

A sizeable flux of SR photons produced by the beam core (primarily in the last dipole) hits the SR masks on either side of the IP (Table 10).

- In NLC, when integrated over the entire bunch train, the flux of SR photons from the core reaches a level that may deserve attention, if only because neither rescattering off mask edges nor multiple photon bounces were taken into account. Their spectrum (Figure 13) is very similar to that of the halo photons on the incoming-beam side (Figure 11).

- In TESLA, about $10^{10}$ core photons/bunch hit the SR mask upstream of the IP, depositing $10^{9} \mathrm{GeV} /$ effective bunch train. Given that the TESLA TPC typically integrates over about 150 bunches, both the halo- and the core-SR flux are cause for serious concern, because the simulations at this stage completely neglect back-scattering and edge-scattering of SR photons off masks and other aperture limitations. It should be noted that the relative intensity of these backgrounds is extremely sensitive to the interrelated aperture settings necessary to simultaneously accommodate an incoming and an outgoing beam (Figure 6). While it is plausible that the effectiveness of the TESLA collimation system may be further improved, these results underscore the urgent need for more detailed studies.

- In CLIC, the flux of intercepted core SR photons is slightly lower than in NLC, presumably due to the fact that the CLIC IR has been optimized for $3 \mathrm{TeV}$ c.m. energy.

\subsection{Loss Patterns and Halo Characterization}

The Appendix regroups additional particle distributions in various locations. These were mostly useful for cross-checking the different programs at an early stage of this study, and are presented here mainly for future reference.

\section{Summary}

Comparative studies of the performance of the post-linac beam-collimation systems in the TESLA, NLC and CLIC linear-collider concepts have shown that the performance of the systems as currently designed is not uniform across projects, and that it does not always meet all the design goals. As of this writing, the CLIC and NLC collimation schemes appear the most promising. But substantial improvements of the TESLA collimation system are expected to result from the ongoing overhaul of their BDS design. Overall, the very existence of an acceptable solution - albeit with the reservations outlined below - suggests that achieving the required performance in future linear colliders is feasible.

It should be pointed out that not all the designs are equally mature: in some areas, substantial uncertainties persist, or realistic performance margins remain to be incorporated.

- The tight aperture settings needed to achieve satisfactory collimation efficiency, coupled with significant beam jitter, can lead to a (possibly unacceptable) luminosity degradation by 
wakefields. Tail-folding octupoles appear very promising to significantly relax the collimation requirements, and more work is clearly warranted here.

- The level of halo SR intercepted near the IR is of some significant concern in the TESLA case, and its implications for detector backgrounds must be thoroughly investigated.

- A careful review of the SR flux produced by the core of the beam is also clearly required for all projects.

- More sophisticated computations of potential SR backgrounds, that include tip-scattering and back-scattering from all aperture limitations, are highly necessary.

- Calculations of the muon flux produced in the collimation and final-focus sections and reaching the IR could not be attempted within the bounds of the present report. Muon-background simulations need to be pursued and updated. The MARS code [12] could be used to crosscheck/validate earlier results.

At a more global level, a recent workshop [13] listed the following questions as most worth pursuing.

\section{- Optics issues.}

- It has been found that good collimation performance is observed in systems with a finalfocus design based on local chromatic corrections. Although not surprising, does this imply that a "traditional" FF design (separate CCS) leaves little room for improvement?

- Can one come to a verifiable conclusion as to whether it is preferable for the betatroncollimation section to precede, or to follow, the energy-collimation module? (This is not only an optics question - issues like fault scenarios are important as well.)

- Impact of machine imperfections. Most simulations to date have considered background and collimation for perfect optics and ideal machines. The case of a "real" machine (i.e. misaligned, imperfectly tuned) should be considered to verify whether there is enough margin for errors. Integrated simulation tools such as MATLIAR [14] potentially can be used to answer some of these questions.

- Halo assumptions. The comparisons presented in this report are based on (hopefully) pessimistic assumptions about the beam halo. Is it possible to predict more accurately and reliably the fractional population of this halo?

\section{References}

[1] Second Report of the International Linear Collider Technical Review Commitee (ILC-TRC), SLAC-R-606, 2003, to be published.

[2] P. Tenenbaum, Collimator Wakefield Calculations for ILC-TRC Report, SLAC, LCC-Note LCC-0101, 2002. 
[3] R. Brinkmann, A. Drozhdin, D. Schulte, M. Seidel, The TESLA Beam Collimation System, DESY Preprint, December 1995, TESLA 95-25.

[4] A. Drozhdin, O. Napoly, N. Walker, TESLA Beam Collimation System Simulations, May 14, 1999, http://www-ap.fnal.gov/ drozhdin/

[5] O. Napoly and N. J. Walker, "TESLA interaction region layout, collimation and extraction," DESY-TESLA-2001-29, Prepared for 5th International Linear Collider Workshop (LCWS 2000), Fermilab, Batavia, Illinois, 24-28 Oct 2000.

[6] P. Raimondi, A. Seryi and P. Tenenbaum, "Tunability of the NLC final focus system," SLAC-PUB-8895, in Proceedings of the IEEE Particle Accelerator Conference (PAC 2001), Chicago, Illinois, 18-22 Jun 2001.

[7] M. Aleksa et al., "CLIC beam delivery system," CLIC-NOTE-551, presented at the 26th Advanced ICFA Beam Dynamics Workshop On Nanometer Size Colliding Beams (Nanobeam 2002), 2-6 Sep 2002, Lausanne, Switzerland.

[8] I. Baishev, A. Drozhdin, and N. Mokhov, 'STRUCT Program User's Reference Manual', SSCL-MAN-0034 (1994), http://www-ap.fnal.gov/ drozhdin/

[9] D. C. Carey, K. L. Brown and C. Iselin, "TURTLE with MAD input (Trace Unlimited Rays Through Lumped Elements): A computer program for simulating charged particle beam transport system and DECAY TURTLE including decay calculations," SLAC-R-544, FERMILAB-PUB-99-232, Sep 1999.

[10] R. Brun, F. Bruyant, M. Maire, A. C. McPherson and P. Zanarini, "Geant3," CERNDD/EE/84-1, Sep 1987.

[11] “TESLA Technical Design Report”, DESY-01-011, March 2001.

[12] N. V. Mokhov, “The MARS code system user's guide version 13(95),” FERMILAB-FN-0628

[13] Collimation Task Force Workshop, SLAC, December 16-18, 2002, http://www-project.slac.stanford.edu/lc/wkshp/colltf2002/

[14] P. Tenenbaum, L. Hendrickson, A. Seryi and G. Stupakov, "Recent developments in the LIAR simulation code," SLAC-PUB-9263, in Proceedings of the 8th European Particle Accelerator Conference (EPAC 2002), Paris, France, 3-7 Jun 2002. 


\section{Collimation-system and beam-halo parameters}

\begin{tabular}{|c||c|c|c||}
\hline parameter & TESLA-500 & NLC-500 & CLIC-500 \\
\hline Center of mass energy E, GeV & 500 & 500 & 500 \\
Number of particles per bunch N & $2 \cdot 10^{10}$ & $0.75 \cdot 10^{10}$ & $0.4 \cdot 10^{10}$ \\
Number of bunches per train & 2820 & 192 & 154 \\
Separation between bunches, ns & 337 & 1.4 & 0.67 \\
Repetition frequency, Hz & 5 & 120 & 200 \\
Average current (each beam), $\mu \mathrm{a}$ & 45.1 & 27.6 & 19.7 \\
Beam power $($ each beam), MW & 11.3 & 6.9 & 4.9 \\
Horiz. normalized emittance $(\sigma), \mathrm{mm} \cdot \mathrm{mrad}$ & 10 & 3.6 & 2.0 \\
Vert. normalized emittance $(\sigma), \mathrm{mm} \cdot \mathrm{mrad}$ & 0.03 & 0.04 & 0.01 \\
Horizontal emittance $(\sigma), \mathrm{mm} \cdot \mathrm{mrad}$ & $2.044 E-05$ & $7.358 E-06$ & $4.088 E-06$ \\
Vertical emittance $(\sigma), \mathrm{mm} \cdot \mathrm{mrad}$ & $6.132 E-08$ & $8.176 E-08$ & $2.044 E-08$ \\
Horizontal beta function in IP, mm & 15.233 & 8 & 10 \\
Vertical beta function in IP, mm & 0.408 & 0.11 & 0.05 \\
Horizontal beam size in IP $(\sigma), \mathrm{nm}$ & 553 & 243 & 202 \\
Vertical beam size in IP $(\sigma), \mathrm{nm}$ & 5 & 3 & 1.5 \\
\hline
\end{tabular}

Table 1: LC-500 beam parameters.

CU'Be COMPOSTE SPOILER CONCEPT $\quad 8 / 17 / 99$

$\begin{array}{ll}\text { depth } & \text { absorption } \\ 50 \mathrm{um} & .21 \mathrm{r.l} \\ 500 \mathrm{um} & .30 \mathrm{r.l} \\ 1000 \mathrm{um} & .39 \mathrm{r.l} .\end{array}$

1000 um $\quad .39$ r.l.

CU PLATE

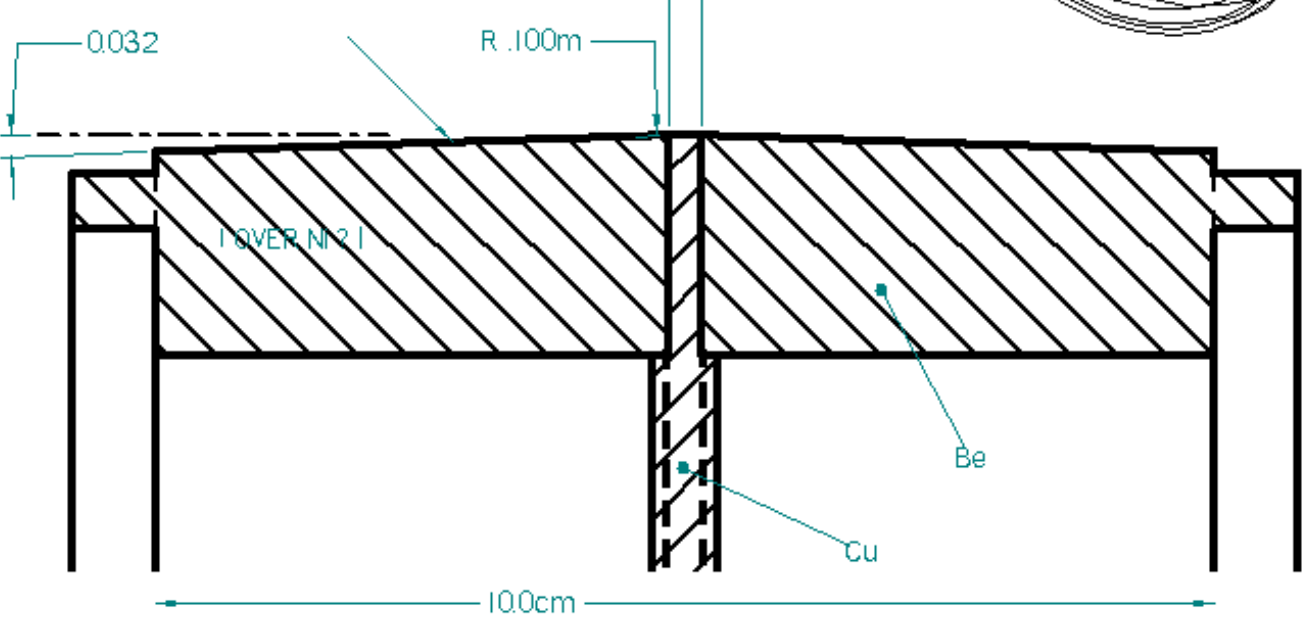

Figure 1: NLC spoiler design. 


\begin{tabular}{|c|c|c|c|c|c|c|c|c|}
\hline & & \multicolumn{3}{|c|}{ Spoilers, absorbers } & \multicolumn{4}{|c|}{ half-aperture } \\
\hline $\mathrm{S}$ & NAME & BETAX & BETAY & DISPERS. & $A_{x}$ & $A_{y}$ & $A_{x}$ & $A_{y}$ \\
\hline $\mathrm{m}$ & & $\mathrm{m}$ & $\mathrm{m}$ & $\mathrm{m}$ & $\mathrm{mm}$ & $\mathrm{mm}$ & $\sigma_{x}$ & $\sigma_{y}$ \\
\hline 666.329 & spo-m2 & 1059 & 326 & -0.113 & 1.50 & 0.67 & 10.3 & 150.0 \\
710.972 & abs-m2 & 25 & 230 & -0.017 & 0.50 & 0.65 & 22.2 & 171.8 \\
777.876 & spoi-1 & 817 & 754 & 0.000 & 1.50 & 0.50 & 11.6 & 74.0 \\
814.916 & abso-1 & 9 & 154 & 0.000 & 0.45 & 0.32 & 33.8 & 107.6 \\
877.557 & spoi-2 & 805 & 894 & 0.000 & 1.50 & 0.50 & 11.7 & 67.3 \\
914.597 & abso-2 & 9 & 183 & 0.000 & 0.30 & 0.32 & 22.5 & 94.7 \\
977.238 & spoi-3 & 825 & 903 & 0.000 & 1.50 & 0.50 & 11.5 & 67.3 \\
1014.278 & abso-3 & 10 & 179 & 0.000 & 0.30 & 0.32 & 21.2 & 94.7 \\
1076.919 & spoi-4 & 822 & 757 & 0.000 & 1.50 & 0.50 & 11.5 & 74.0 \\
1105.145 & abso-4 & 250 & 1045 & 0.000 & 1.57 & 0.77 & 21.9 & 96.6 \\
1183.369 & spoiX1 & 1127 & 220 & 0.034 & 2.00 & 0.28 & 13.2 & 77.0 \\
1207.469 & absoX1 & 203 & 471 & 0.017 & 1.80 & 0.70 & 28.1 & 130.0 \\
1269.269 & absX1a & 722 & 295 & 0.029 & 2.50 & 0.50 & 21.0 & 119.0 \\
1275.769 & spoiX2 & 1172 & 214 & 0.036 & 2.00 & 0.28 & 12.9 & 77.0 \\
1299.469 & absoX2 & 200 & 465 & 0.012 & 1.80 & 0.70 & 28.1 & 130.0 \\
1367.769 & spoiY1 & 53 & 4691 & -0.013 & 0.42 & 1.31 & 13.0 & 77.0 \\
1391.469 & absoY1 & 132 & 825 & -0.036 & 4.40 & 1.90 & 85.1 & 265.0 \\
1459.769 & spoiY2 & 52 & 4681 & -0.017 & 0.42 & 1.31 & 13.0 & 77.0 \\
1526.393 & DUMP3a & 78 & 273 & -0.017 & 8.00 & 8.00 & 200 & 2000 \\
1598.933 & DUMP3b & 2006 & 7199 & -0.017 & 8.00 & 8.00 & 40.0 & 380.0 \\
1636.393 & DUMP2 & 1748 & 21488 & -0.017 & 55.0 & 55.0 & 291 & 1528 \\
1748.393 & DUMP1 & 7620 & 12200 & -0.017 & 10.0 & 10.0 & 25.3 & 370 \\
\hline 1766.393 & IP & & & & & & & \\
\hline
\end{tabular}

Table 2: Horizontal and vertical $\beta$-functions, dispersion, and apertures at the spoilers and absorbers in TESLA. Off-momentum spoiler spo-m 2 is at $\mathrm{dP} / \mathrm{P}=0.015$. The betatron spoilers spoi-1, spoi2, spoi-3, spoi-4 are at $A_{x}=11.6 \sigma_{x}$ and $A_{y}=74 \sigma_{y}$. The absorbers of these spoilers are at $A_{x}=21-26 \sigma_{x}$ and $A_{y}=94-108 \sigma_{y}$ to eliminate losses of primary particles at the absorbers. Spoilers of the second stage of collimation are at $A_{x}=13 \sigma_{x}$ and $A_{y}=77 \sigma_{y}$ in the horizontal and vertical plane. Absorber apertures are chosen by beam tracking to eliminate losses of primary particles at the absorbers. The beam halo is represented by $5 \cdot 10^{5}$ rays with $1 / x$ and $1 / y$ density distributions for amplitudes of $A_{x}=(7-18) \sigma_{x}$ and $A_{y}=(40-120) \sigma_{y}$, and with a momentum spread of $\sigma(d P / P)=1 \%$. DUMP3b is not used in the current simulations. If it were, it would decrease photon losses in DUMP1 by a factor of 3.2. 


\begin{tabular}{|c|c|c|c|c|c|c|c|c|}
\hline & & \multicolumn{3}{|c|}{ spoilers, absorbers } & \multicolumn{4}{|c|}{ half-aperture } \\
\hline $\mathrm{S}$ & Name & BetaX & BetaY & Dispers. & $A_{x}$ & $A_{y}$ & $A_{x}$ & $A_{y}$ \\
\hline $\mathrm{m}$ & & $\mathrm{m}$ & $\mathrm{m}$ & $\mathrm{m}$ & $\mathrm{mm}$ & $\mathrm{mm}$ & $\sigma_{x}$ & $\sigma_{y}$ \\
\hline 0.007 & SP1 & 35.83 & 7.07 & 0.000 & 0.30 & 0.25 & 18.5 & 326 \\
\hline 76.491 & SP2 & 103.28 & 523.42 & 0.000 & 0.28 & 0.20 & 10.2 & 31 \\
\hline 152.374 & AB3 & 35.82 & 7.08 & 0.000 & 1.00 & 1.00 & 61.5 & 1304 \\
\hline 152.491 & SP3 & 35.82 & 7.08 & 0.000 & 0.30 & 0.25 & 18.5 & 326 \\
\hline 228.374 & $\mathrm{AB} 4$ & 103.28 & 523.42 & 0.000 & 1.00 & 1.00 & 36.3 & 153 \\
\hline 228.491 & SP4 & 103.28 & 523.42 & 0.000 & 0.28 & 0.20 & 10.2 & 31 \\
\hline 288.866 & AB5 & 59.74 & 5.36 & 0.000 & 1.40 & 1.00 & 66.8 & 1500 \\
\hline 288.983 & SP5 & 59.74 & 5.36 & 0.000 & 0.42 & 0.25 & 20.0 & 375 \\
\hline 497.592 & SPE & 226.69 & 10058.96 & 0.213 & 3.20 & 3.20 & 78.3 & 112 \\
\hline 662.449 & $\mathrm{ABEa}$ & 244.35 & 329.16 & 0.007 & 1.10 & 1.10 & 25.9 & 212 \\
\hline 664.749 & $\mathrm{ABEb}$ & 240.00 & 283.52 & 0.006 & 1.10 & 1.10 & 26.2 & 228 \\
\hline 890.421 & AB10 & 13276.75 & 149854.87 & 0.000 & 4.40 & 4.40 & 14.1 & 40 \\
\hline 911.000 & AB9 & 38123.55 & 55295.79 & 0.000 & 6.50 & 3.00 & 12.3 & 45 \\
\hline 984.952 & AB7 & 36.63 & 82.44 & -0.026 & 3.90 & 1.00 & 238 & 385 \\
\hline 1384.005 & DUMP1 & 21712.01 & 30406.34 & -0.115 & 8.00 & 20.00 & 20 & 400 \\
\hline 1420.795 & DUMP2 & 33628.04 & 52550.49 & -0.115 & 8.50 & 20.00 & 17.1 & 303 \\
\hline 1433.815 & IP & & & & & & & \\
\hline
\end{tabular}

Table 3: Horizontal and vertical $\beta$-functions, dispersion and apertures at the spoilers and absorbers in NLC. The off-energy spoiler SPE is at $\mathrm{dP} / \mathrm{P}=0.015$ for $A_{x}=0$. Betatron spoilers SP2 and SP4 are at $A_{x}=10 \sigma_{x}$ and $A_{y}=31 \sigma_{y}$. The beam halo is represented by $5 \cdot 10^{5}$ rays with $1 / x$ and $1 / y$ density distributions for amplitudes of $A_{x}=(6-16) \sigma_{x}$ and $A_{y}=(24-73) \sigma_{y}$, and with a momentum spread of $\sigma(d P / P)=1 \%$.

\begin{tabular}{|c|c|c|c|c|c|c|c|c|}
\hline & & \multicolumn{3}{|c|}{ spoilers, absorbers } & \multicolumn{4}{|c|}{ half-aperture } \\
\hline $\mathrm{S}$ & Name & BetaX & BetaY & Dispers. & $A_{x}$ & $A_{y}$ & $A_{x}$ & $A_{y}$ \\
\hline $\mathrm{m}$ & & $\mathrm{m}$ & $\mathrm{m}$ & $\mathrm{m}$ & $\mathrm{mm}$ & $\mathrm{mm}$ & $\sigma_{x}$ & $\sigma_{y}$ \\
\hline 549.687 & ENGYSP & 1405 & 71352 & 0.324 & 1.60 & 4.00 & 21 & 105 \\
714.688 & ENGYAB & 3212 & 39647 & 0.500 & 3.00 & 4.00 & 26 & 141 \\
879.902 & ENGYAB2 & 1405 & 71412 & 0.324 & 2.00 & 4.60 & 26 & 120 \\
1353.438 & YSP0 & 361 & 467 & 0.000 & 0.34 & 0.20 & 8.9 & 65 \\
1473.462 & YSP1 & 114 & 493 & 0.000 & 0.30 & 0.20 & 14.3 & 65 \\
1489.284 & XSP1 & 270 & 103 & 0.000 & 0.30 & 0.20 & 8.9 & 142 \\
1586.305 & YSP2 & 114 & 495 & 0.000 & 0.30 & 0.20 & 14.3 & 65 \\
1602.127 & XSP2 & 270 & 105 & 0.000 & 0.30 & 0.20 & 8.9 & 142 \\
1912.723 & ABS3 & 209 & 204 & 0.000 & 0.45 & 0.24 & 15.5 & 120 \\
2515.997 & DUMP1 & 71438 & 155188 & -0.204 & 7.50 & 6.00 & 13.9 & 106 \\
2521.497 & DUMP2 & 76677 & 165979 & -0.204 & 7.50 & 6.00 & 13.4 & 103 \\
\hline 2544.74 & IP & & \multicolumn{9}{|c|c|c|c|c|}{} \\
\hline
\end{tabular}

Table 4: Horizontal and vertical $\beta$-functions, dispersion and apertures at the spoilers and absorbers in CLIC. The off-energy spoiler ENGYSP is at $\mathrm{dP} / \mathrm{P}=0.005$; off-energy absorbers ENGYAB, EN$\mathrm{GYAB} 2$ are at $\mathrm{dP} / \mathrm{P}=0.006$. The betatron spoilers are at $A_{x}=8.9 \sigma_{x}$ and $A_{y}=65 \sigma_{y}$; photon dumps DUMP1 and DUMP2 are at $A_{x} \approx 13.5 \sigma_{x}$ and $A_{y}=103 \sigma_{y}$. The beam halo is represented by $5 \cdot 10^{5}$ rays with $1 / x$ and $1 / y$ density distributions for amplitudes of $A_{x}=(5.7-14.2) \sigma_{x}$ and $A_{y}=(54-162) \sigma_{y}$, and with a momentum spread of $\sigma(d P / P)=1 \%$. 


\begin{tabular}{|c|c|c|c|c|c|c|c|c|c|c|c|}
\hline \multirow[t]{2}{*}{ name } & \multicolumn{2}{|c|}{ length } & \multirow{2}{*}{$\begin{array}{c}\text { mate- } \\
\text { rial }\end{array}$} & \multirow[t]{2}{*}{ name } & \multicolumn{2}{|c|}{ length } & \multirow{2}{*}{$\begin{array}{c}\text { mate- } \\
\text { rial }\end{array}$} & \multirow[t]{2}{*}{ name } & \multicolumn{2}{|c|}{ length } & \multirow{2}{*}{$\begin{array}{c}\text { mate- } \\
\text { rial }\end{array}$} \\
\hline & $\mathrm{mm}$ & r.l. & & & $\mathrm{mm}$ & r.l. & & & $\mathrm{mm}$ & r.l. & \\
\hline \multicolumn{4}{|c|}{ NLC } & \multicolumn{4}{|c|}{ TESLA } & \multicolumn{4}{|c|}{ CLIC } \\
\hline SP1 & 117.15 & $0.5+0.3$ & $\mathrm{Cu}+\mathrm{Be}$ & spo-m2 & 35 & 1 & $\mathrm{Ti}$ & ENGYSP & 94 & 0.5 & $\mathrm{C}$ \\
\hline SP2 & 117.15 & $0.5+0.3$ & $\mathrm{Cu}+\mathrm{Be}$ & abs-m2 & 500 & 35 & $\mathrm{Cu}$ & ENGYAB & 429 & 30 & $\mathrm{Cu}$ \\
\hline AB3 & 429 & 30 & $\mathrm{Cu}$ & spoi-1 & 35 & 1 & $\mathrm{Ti}$ & ENGYAB2 & 429 & 30 & $\mathrm{Cu}$ \\
\hline SP3 & 117.15 & $0.5+0.3$ & $\mathrm{Cu}+\mathrm{Be}$ & abso-1 & 500 & 35 & $\mathrm{Cu}$ & YSP0 & 117.15 & $0.5+0.3$ & $\mathrm{Cu}+\mathrm{Be}$ \\
\hline $\mathrm{AB} 4$ & 429 & 30 & $\mathrm{Cu}$ & spoi-2 & 35 & 1 & $\mathrm{Ti}$ & $\mathrm{ABS} 0(*)$ & 429 & 30 & $\mathrm{Cu}$ \\
\hline SP4 & 117.15 & $0.5+0.3$ & $\mathrm{Cu}+\mathrm{Be}$ & abso-2 & 500 & 35 & $\mathrm{Cu}$ & YSP1 & 117.15 & $0.5+0.3$ & $\mathrm{Cu}+\mathrm{Be}$ \\
\hline AB5 & 429 & 30 & $\mathrm{Cu}$ & spoi-3 & 35 & 1 & $\mathrm{Ti}$ & XSP1 & 117.15 & $0.5+0.3$ & $\mathrm{Cu}+\mathrm{Be}$ \\
\hline SP5 & 117.15 & $0.5+0.3$ & $\mathrm{Cu}+\mathrm{Be}$ & abso-3 & 500 & 35 & $\mathrm{Cu}$ & $\operatorname{ABS} 1(*)$ & 429 & 30 & $\mathrm{Cu}$ \\
\hline SPE & 17.8 & 0.5 & $\mathrm{Ti}$ & spoi-4 & 35 & 1 & $\mathrm{Ti}$ & $\mathrm{ABS} 1 \mathrm{a}(*)$ & 429 & 30 & $\mathrm{Cu}$ \\
\hline $\mathrm{ABEa}$ & 214.5 & 15 & $\mathrm{Cu}$ & abso-4 & 500 & 35 & $\mathrm{Cu}$ & YSP2 & 117.15 & $0.5+0.3$ & $\mathrm{Cu}+\mathrm{Be}$ \\
\hline $\mathrm{ABEb}$ & 214.5 & 15 & $\mathrm{Cu}$ & spoiX1 & 35 & 1 & $\mathrm{Ti}$ & XSP2 & 117.15 & $0.5+0.3$ & $\mathrm{Cu}+\mathrm{Be}$ \\
\hline AB10 & 429 & 30 & $\mathrm{Cu}$ & absoX1 & 500 & 35 & $\mathrm{Cu}$ & $\operatorname{ABS} 2(*)$ & 429 & 30 & $\mathrm{Cu}$ \\
\hline AB9 & 429 & 30 & $\mathrm{Cu}$ & absX1a & 500 & 35 & $\mathrm{Cu}$ & $\mathrm{ABS} 2 \mathrm{a}(*)$ & 429 & 30 & $\mathrm{Cu}$ \\
\hline \multirow[t]{5}{*}{ AB7 } & 214.5 & 15 & $\mathrm{Cu}$ & spoiX2 & 35 & 1 & $\mathrm{Ti}$ & YSP3(*) & 117.15 & $0.5+0.3$ & $\mathrm{Cu}+\mathrm{Be}$ \\
\hline & & & & absoX2 & 500 & 35 & $\mathrm{Cu}$ & XSP3(*) & 117.15 & $0.5+0.3$ & $\mathrm{Cu}+\mathrm{Be}$ \\
\hline & & & & spoiY1 & 35 & 1 & $\mathrm{Ti}$ & YSP4(*) & 117.15 & $0.5+0.3$ & $\mathrm{Cu}+\mathrm{Be}$ \\
\hline & & & & absoY1 & 500 & 35 & $\mathrm{Cu}$ & XSP4(*) & 117.15 & $0.5+0.3$ & $\mathrm{Cu}+\mathrm{Be}$ \\
\hline & & & & spoiY2 & 35 & 1 & $\mathrm{Ti}$ & ABS3 & 429 & 30 & $\mathrm{Cu}$ \\
\hline
\end{tabular}

Table 5: Collimator length and material. Collimators with $(*)$ are not used in the calculations because they do not affect the collimation efficiency.

\begin{tabular}{|c||c|c|c||}
\hline & TESLA & NLC & CLIC \\
\hline Range of $A_{x} / \sigma_{x}$ & $7-18$ & $6-16$ & $5.7-14.2$ \\
Range of $A_{y} / \sigma_{y}$ & $40-120$ & $24-73$ & $54-162$ \\
Momentum spread $\sigma(d P / P), \%$ & 1 & 1 & 1 \\
Typical number of rays & $5 \cdot 10^{5}$ & $5 \cdot 10^{5}$ & $5 \cdot 10^{5}$ \\
\hline
\end{tabular}

Table 6: Halo parameters used in simulations. The halo was represented by rays distributed in phase space in the shape of a ring with $1 / x$ and $1 / y$ density distribution and covering a machinedependent range of amplitudes $A_{x}$ and $A_{y}$ (appropriately overlapping the nominal collimation depth), and with a sizeable energy spread. 

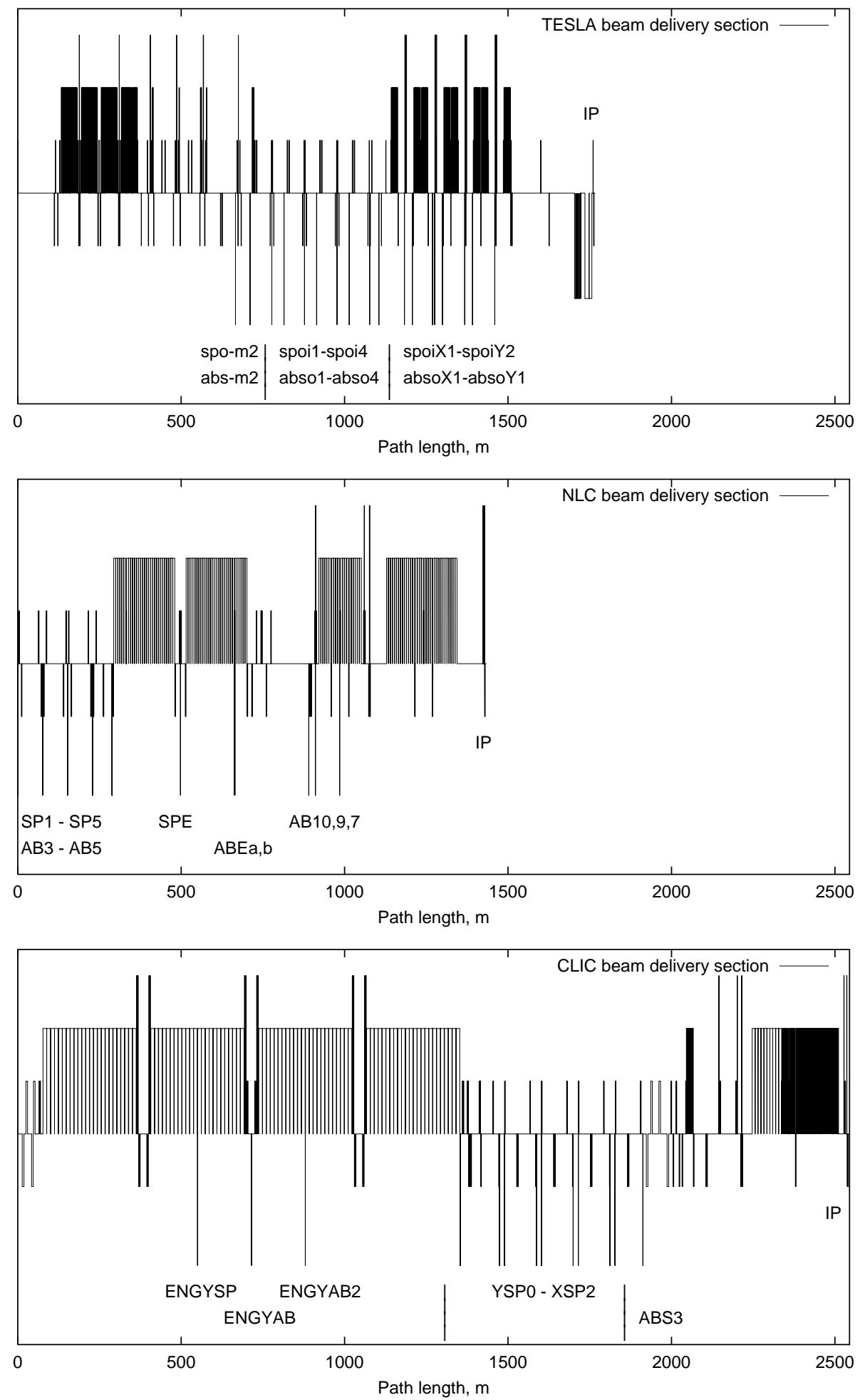

Figure 2: Collimator locations in the TESLA (top), NLC (middle) and CLIC (bottom) beam delivery systems. 

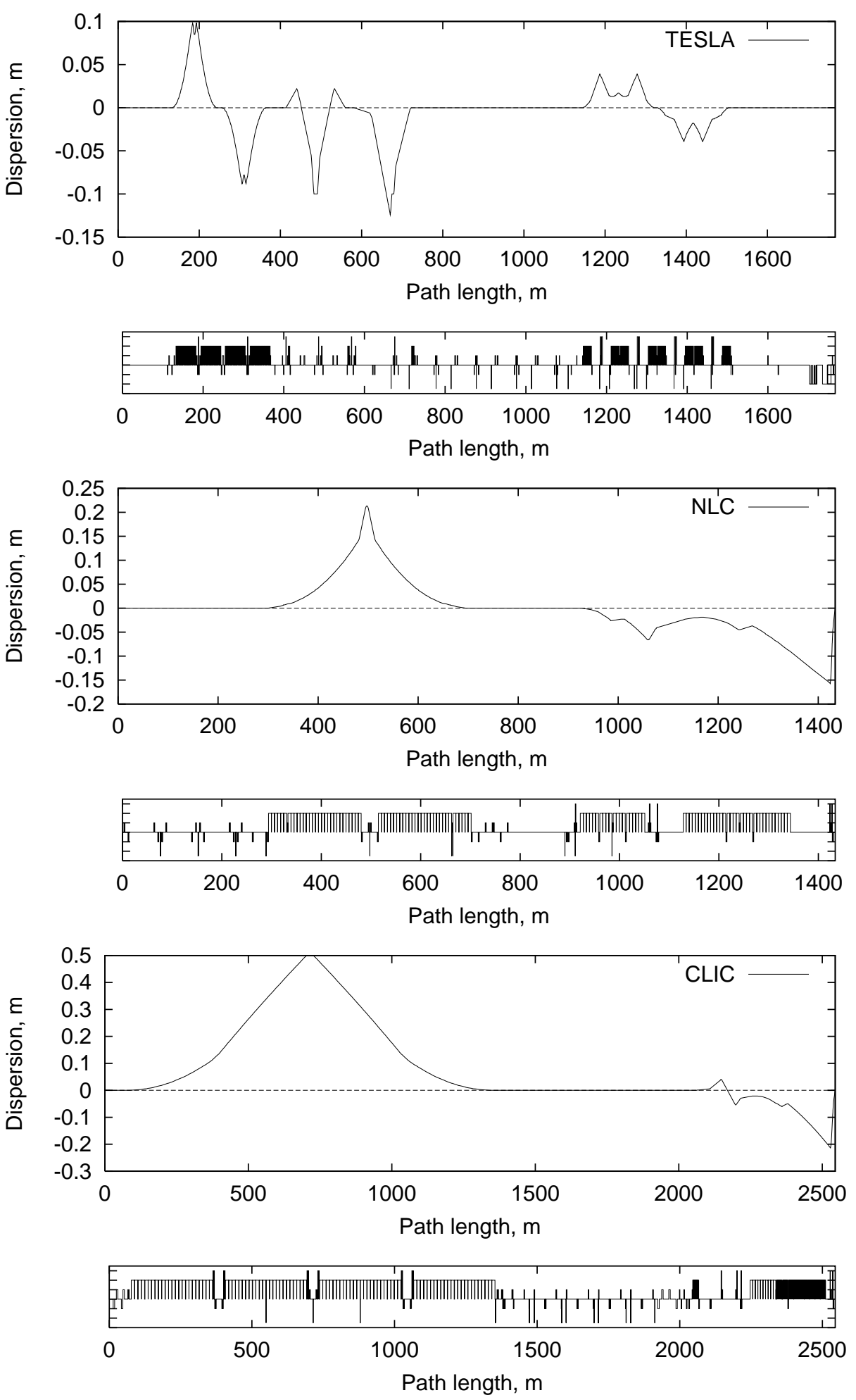

Figure 3: Horizontal dispersion in the TESLA (top), NLC (middle) and CLIC (bottom) beam delivery systems. 

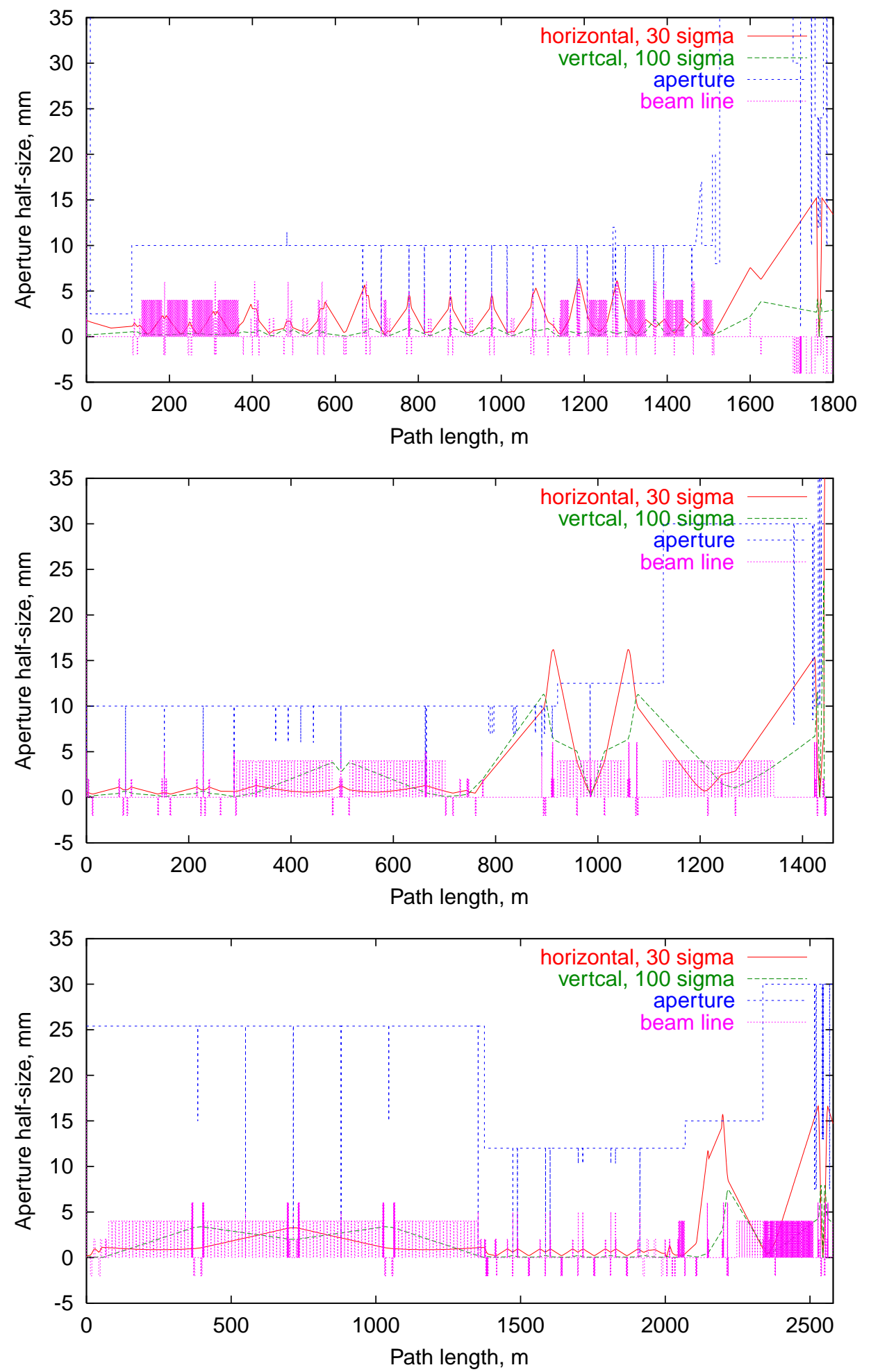

Figure 4: Beam size (for $30 \sigma_{x}$ and $100 \sigma_{y}$ incoming beam) and apertures in the TESLA (top), NLC (middle) and CLIC (bottom) beam delivery systems. 

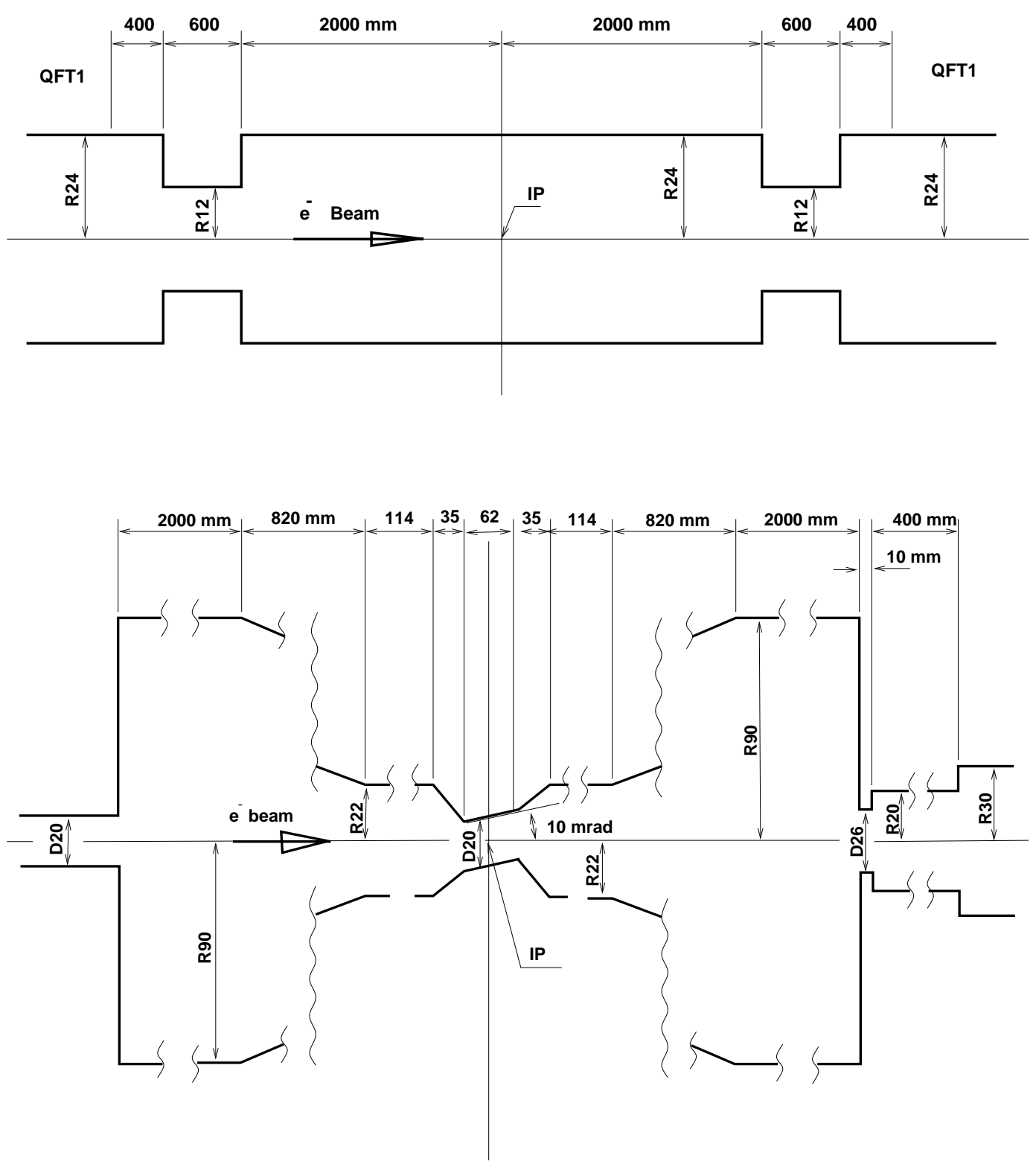

Figure 5: Aperture map in the IP region for TESLA (top) and NLC (bottom), as modelled in the simulations for this report. Note the TESLA vertex detector should have been simulated by a $15 \mathrm{~mm}$-radius aperture limitation. 

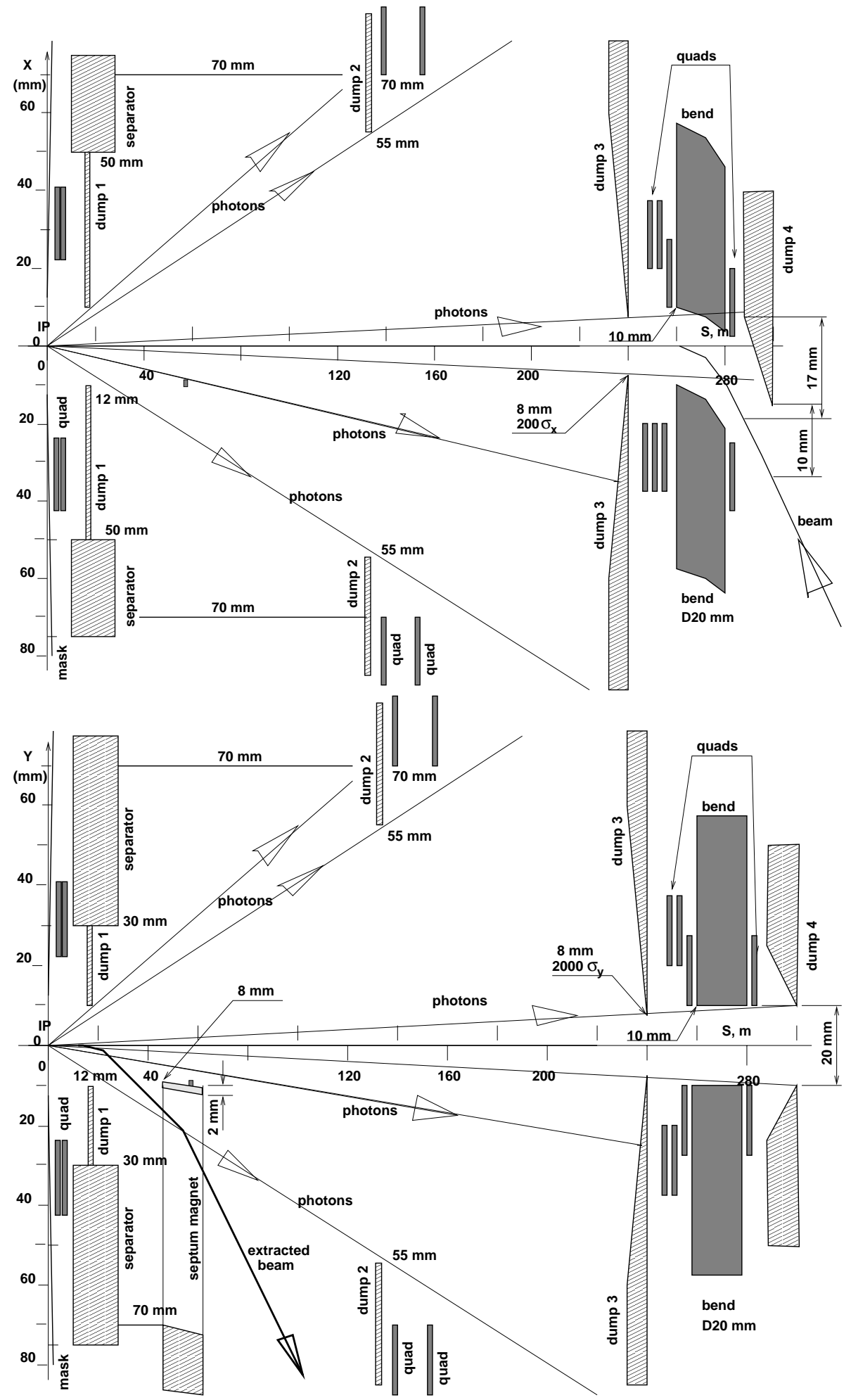

Figure 6: TESLA-500 beamstrahlung collimation. 

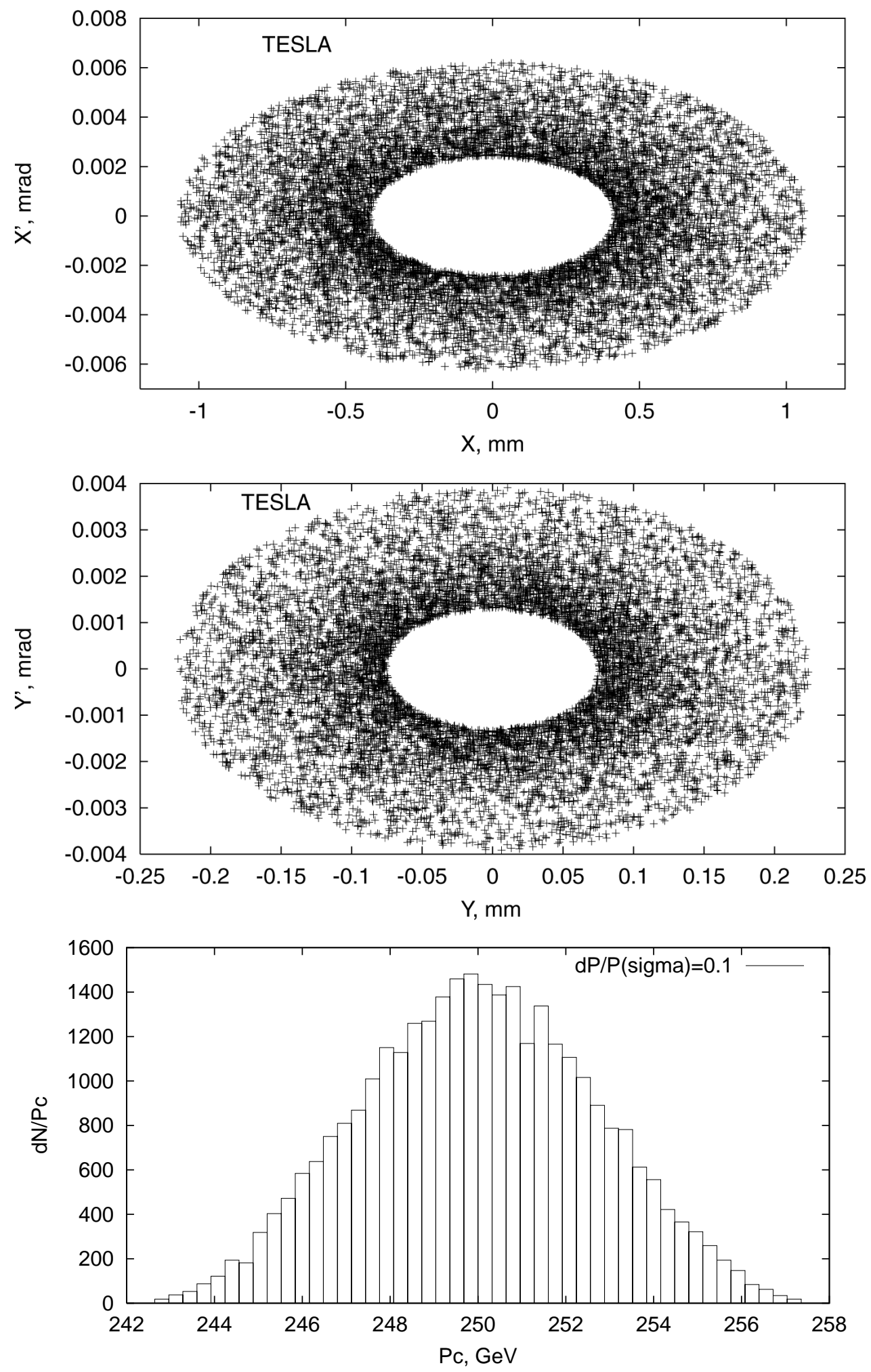

Figure 7: Initial particle population in the phase plane at the BDS entrance and energy distribution used for modelling the beam halo in TESLA. This halo is represented by $5 \cdot 10^{5}$ rays with $1 / x$ and $1 / y$ density distributions for amplitudes of $A_{x}=(7-18) \sigma_{x}$ and $A_{y}=(40-120) \sigma_{y}$, and with a momentum spread of $\sigma(d P / P)=1 \%$. 

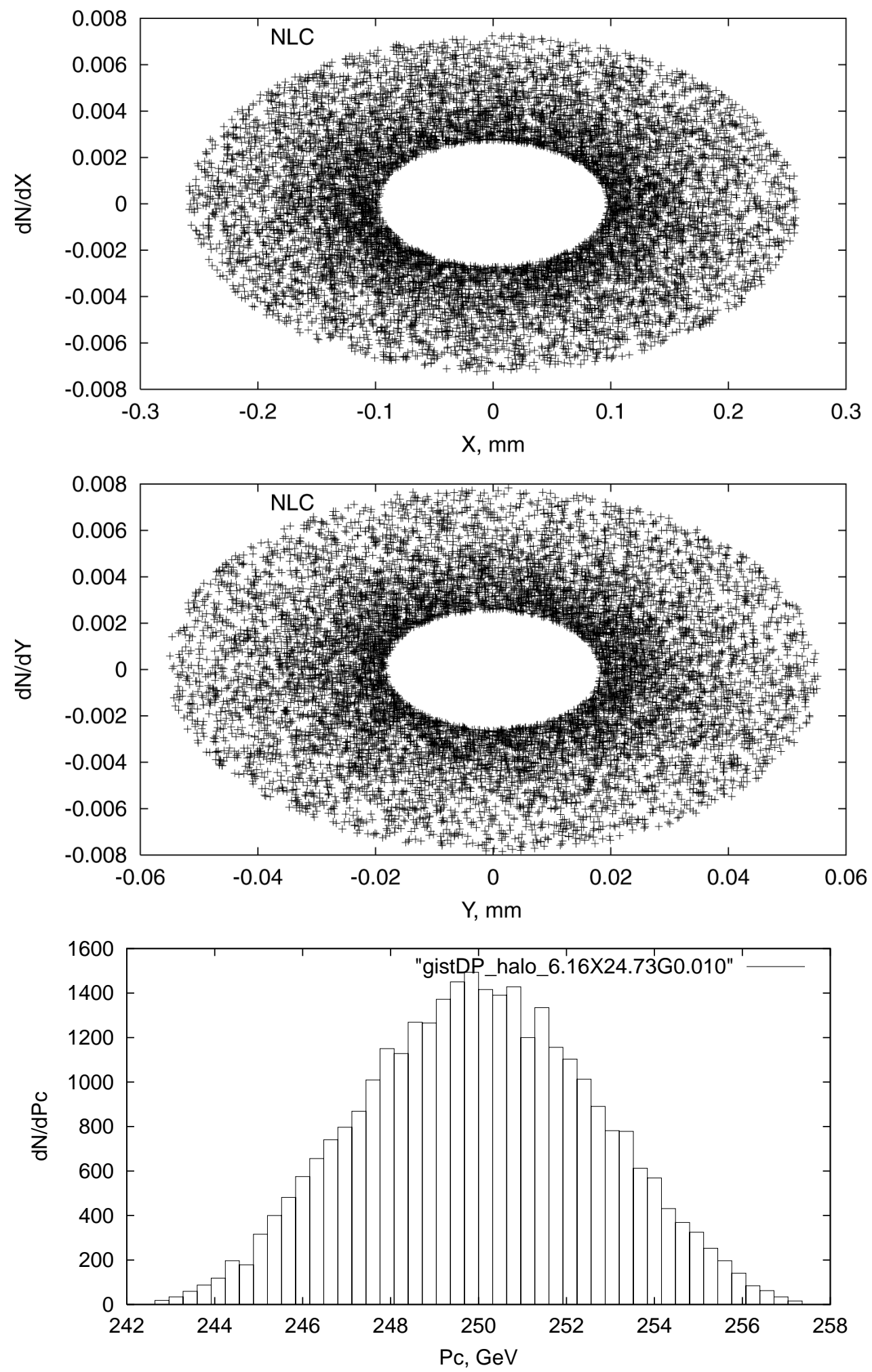

Figure 8: Initial particle population in the phase plane at the BDS entrance and energy distribution used for modelling the beam halo in NLC. This halo is represented by $5 \cdot 10^{5}$ rays with $1 / x$ and $1 / y$ density distributions for amplitudes of $A_{x}=(6-16) \sigma_{x}$ and $A_{y}=(24-73) \sigma_{y}$, and with a momentum spread of $\sigma(d P / P)=1 \%$. 

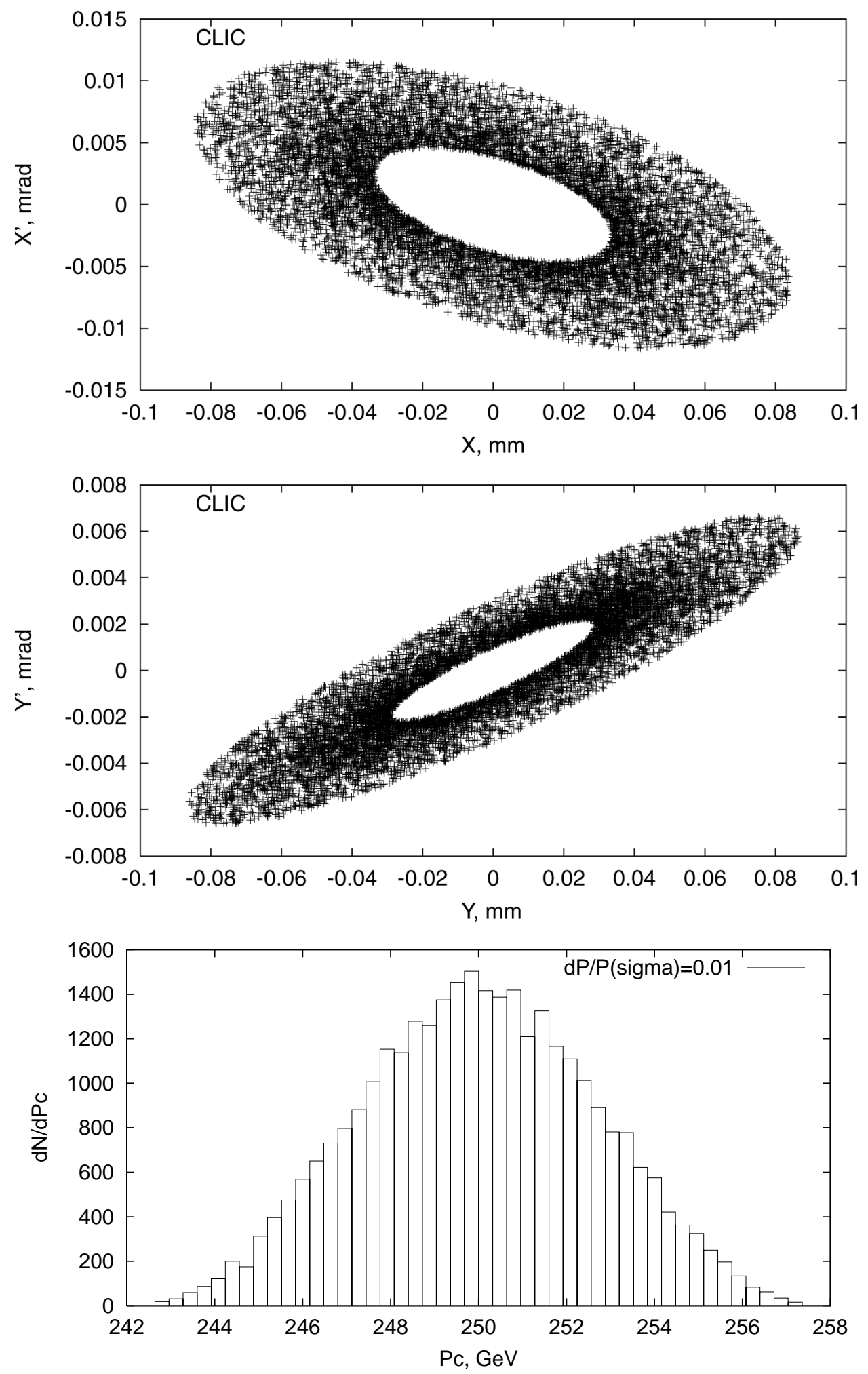

Figure 9: Initial particle population in the phase plane at the BDS entrance and energy distribution used for modelling the beam halo in CLIC. This halo is represented by $5 \cdot 10^{5}$ rays with $1 / x$ and $1 / y$ density distributions for amplitudes of $A_{x}=(5.7-14.2) \sigma_{x}$ and $A_{y}=(54-162) \sigma_{y}$, and with a momentum spread of $\sigma(d P / P)=1 \%$. 


\section{Main Comparative Results}

\begin{tabular}{llccc}
\hline & & TESLA & JLC-X/NLC & CLIC \\
\hline \hline Nominal collimation depth & $\# \sigma_{x, y}^{\beta}$ at spoiler & 12,74 & 10,31 & 9,65 \\
\hline Energy collimator & $\mathrm{x}$ gap $[\mathrm{mm}]$ & \pm 1.50 & \pm 3.20 & \pm 1.60 \\
& $\sigma_{x, y}[\mu \mathrm{m}]$ & $154,4.5$ & 534,29 & 814,38 \\
\hline Betatron collimator & & & & \\
Final-doublet phase & $\mathrm{x}, \mathrm{y}$ gaps $[\mathrm{mm}]$ & $\pm 1.50, \pm 0.50$ & $\pm 0.30, \pm 0.20$ & $\pm 0.34, \pm 0.20$ \\
& $\sigma_{x, y}[\mu \mathrm{m}]$ & 129,7 & $28,6.5$ & 38,3 \\
IP phase & $\mathrm{x}, \mathrm{y}$ gaps [mm] & $\pm 1.50, \pm 0.50$ & $\pm 0.30, \pm 0.25$ & $\pm 0.30, \pm 0.20$ \\
& $\sigma_{x, y}[\mu \mathrm{m}]$ & 128,7 & $16,0.8$ & 22,3 \\
\hline Primary-collimation efficiency & & 0.01 & $<1 \times 10^{-5}$ & $<3 \times 10^{-4}$ \\
\hline Losses in sec. collim. section & $\mathrm{particles} / \mathrm{bunch}$ & $2.4 \times 10^{5}$ & 50 & 1000 \\
\hline \hline Effective collimation depth & $\# \sigma_{x, y}^{\beta}$ at FD & 13,80 & 15,31 & 11,100 \\
\hline
\end{tabular}

Table 7: Main parameters of the post-linac primary collimation systems, as excerpted from Tables 2-4. $\sigma_{x, y}$ are the horizontal and vertical beam size at the primary spoiler (including the dispersive contribution); $\sigma_{x, y}^{\beta}$ refer to the betatron contributions alone. In some cases, the spoiler settings must be tighter than the effective collimation depth (at the final doublet) because of dispersive or higher-order effects.
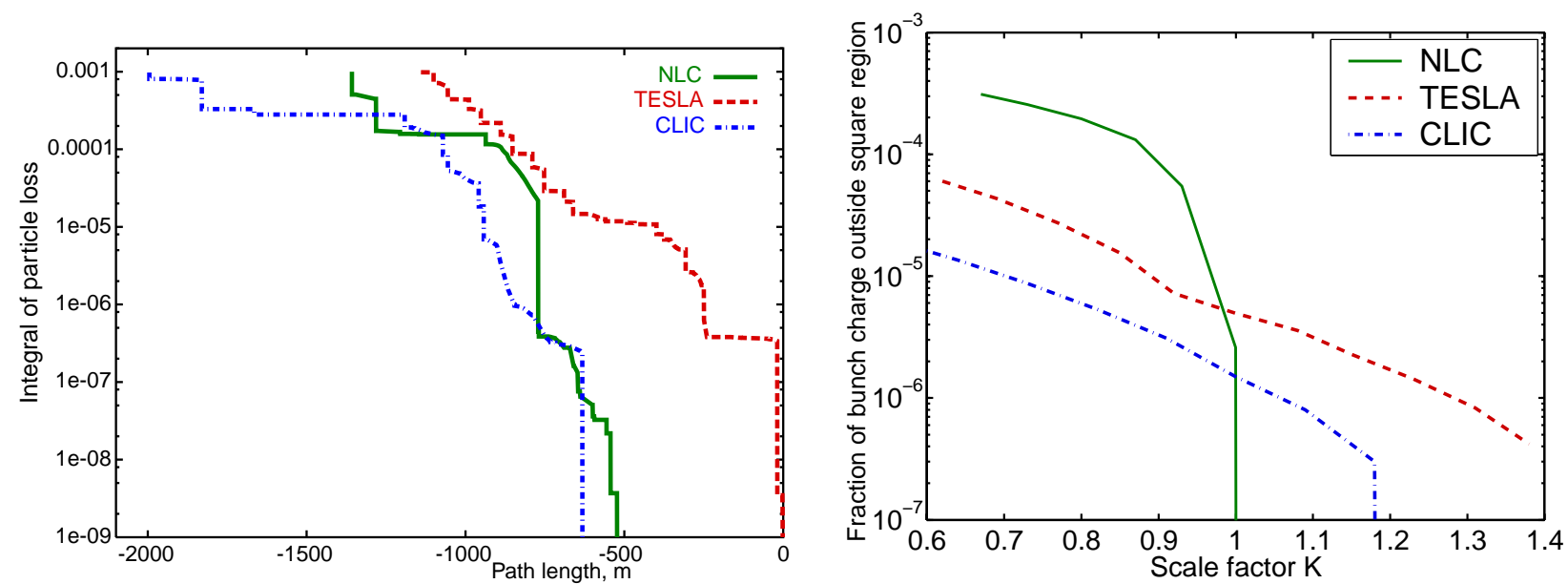

Figure 10: Collimation-system performance assuming an incident fractional halo of $10^{-3}$. Left: fractional loss of charged-halo particles, integrating back, starting at the IP, and normalized to the nominal bunch charge. The horizontal scale shows the distance from the IP. The upstream edge of the secondary-collimation system is located at -543 and $-583 \mathrm{~m}$ in NLC and TESLA respectively. In CLIC, the last betatron absorber is located at $-632 \mathrm{~m}$. Right: number of charged-halo particles per bunch, normalized to the nominal bunch charge, in a rectangular $x-y$ window at the entrance to the final doublet, as a function of the collimation depth. The scale factor $K$ defines the window dimension: for $K=1$, the window size corresponds to the effective collimation depth listed, for each machine, in Table 7. 


\begin{tabular}{|c|c|c|c|}
\hline & TESLA & NLC & CLIC-500 \\
\hline \# bunches /(effective train) & 150 & 192 & 154 \\
\hline \multicolumn{4}{|c|}{ Losses on SR mask upstream of FD } \\
\hline Mean photon energy $(\mathrm{MeV})$ & 0.474 & 0.031 & 0.032 \\
\hline \# photons/bunch & $1.41 \cdot 10^{6}$ & $4.52 \cdot 10^{5}$ & $8.5 \cdot 10^{3}$ \\
\hline /eff. train & $2.11 \cdot 10^{8}$ & $8.68 \cdot 10^{7}$ & $1.3 \cdot 10^{6}$ \\
\hline \multicolumn{4}{|l|}{ Total photon energy $(\mathrm{GeV})$} \\
\hline /bunch & 670 & 14 & 0.28 \\
\hline /eff. train & $1.00 \cdot 10^{5}$ & 2700 & 43 \\
\hline Charged halo (particles/bunch) & 7440 & (none) & (none) \\
\hline \multicolumn{4}{|c|}{ Losses on SR mask downstream of outgoing-side FD } \\
\hline Mean photon energy $(\mathrm{MeV})$ & 10.1 & - & - \\
\hline \# photons/bunch & $1.17 \cdot 10^{7}$ & - & - \\
\hline /eff. train & $1.75 \cdot 10^{9}$ & - & - \\
\hline Total photon energy $(\mathrm{GeV})$ & & & \\
\hline /bunch & $1.17 \cdot 10^{5}$ & - & - \\
\hline /eff. train & $1.75 \cdot 10^{7}$ & - & - \\
\hline Charged halo (particles/bunch) & 246 & - & - \\
\hline
\end{tabular}

Table 8: Synchrotron radiation from the beam halo hitting IR SR masks. The photon energy spectrum is displayed in Figure 11. The losses tabulated refer to masks DUMP1 for TESLA and DUMP2 for NLC and CLIC of the corresponding beam line. The number of bunches per 'effective' train reflects the sensitivity window of the TPC. It is equal to 150 bunches $(50 \mu \mathrm{s})$ for TESLA, and to the nominal number of bunches per train for NLC and CLIC.

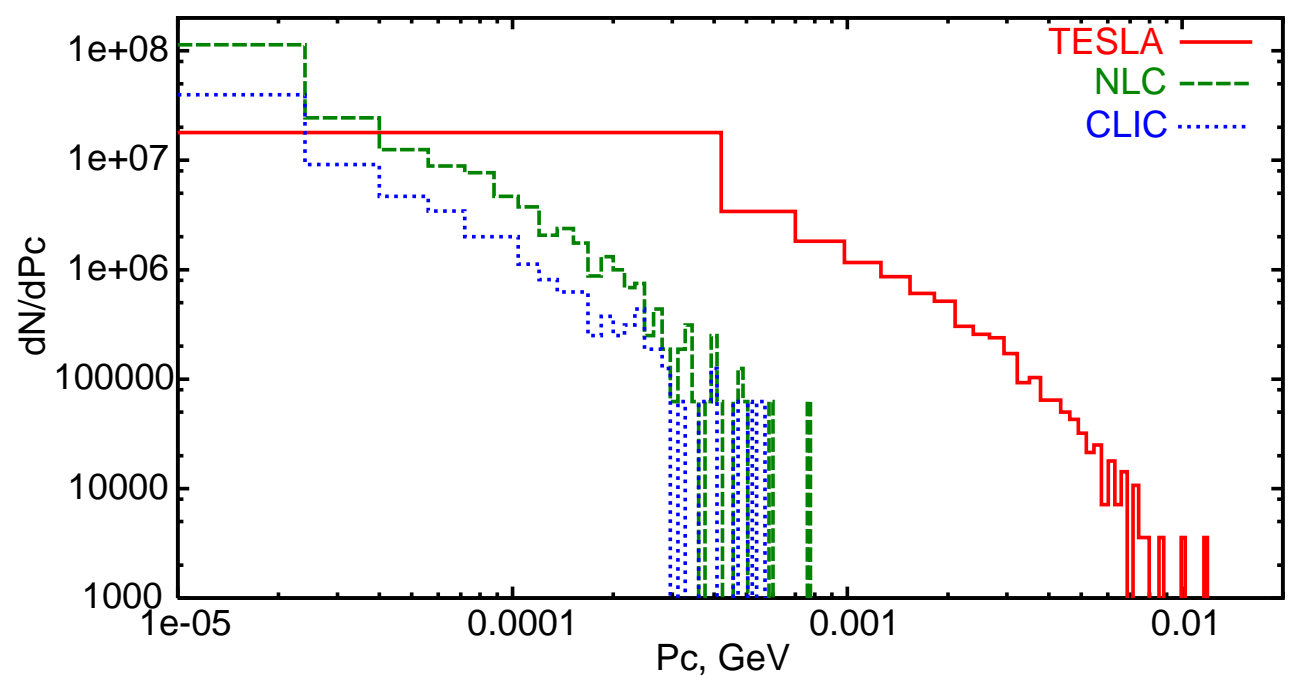

Figure 11: Energy spectrum of synchrotron-radiation photons from the beam halo, that hit DUMP1 (for TESLA) or DUMP2 (for NLC and CLIC). (The normalization of the vertical scale is arbitrary and is different for each machine.) 


\begin{tabular}{llccc}
\hline & & TESLA & JLC-X/NLC & CLIC \\
\hline \hline Upstream detector mask & & & & \\
Radius & $\mathrm{mm}$ & 12 & 10 (QD0) & 13 \\
Halo photon losses & $\mathrm{mW}$ & 0.03 & 0 & $1.8 \times 10^{-6}$ \\
& $\mathrm{GeV} / \mathrm{bunch}$ & 13 & 0 & $3.8 \times 10^{-4}$ \\
\hline Vertex detector & $\mathrm{mm}$ & - & 10 & \\
Radius & $\mathrm{mW}$ & - & $<10^{-7}$ & $1.6 \times 10^{-3}$ \\
Halo photon losses & $\mathrm{GeV} / \mathrm{bunch}$ & - & $<2.7 \times 10^{-5}$ & 0.33 \\
& & & & \\
\hline Downstream detector mask & $\mathrm{mm}$ & 12 & 13 (lum. monitor) & 13 \\
Radius & $\mathrm{mW}$ & 0.36 & 0 & 0.011 \\
Halo photon losses & $\mathrm{GeV} /$ bunch & 158 & 0 & 2.2 \\
& & & &
\end{tabular}

Table 9: Synchrotron-radiation losses from beam halo near the IP. The TESLA vertex detector was not included in the simulation (see Figure 5).

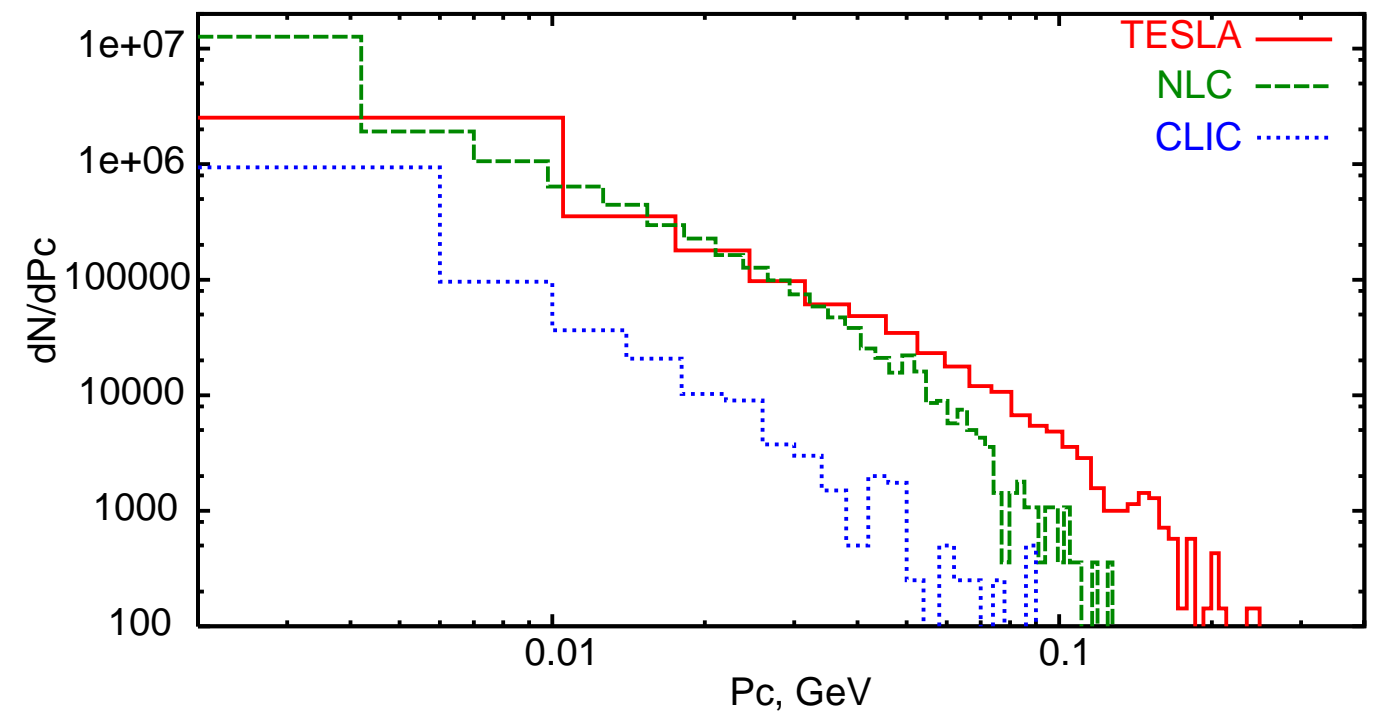

Figure 12: Energy spectrum of all halo-induced SR photons crossing a scoring plane at the IP. TESLA: $1.58 \cdot 10^{7}$ photons/bunch, $1.17 \cdot 10^{5} \mathrm{GeV} /$ bunch, mean photon energy $=7.40 \mathrm{MeV}$. NLC: $1.91 \cdot 10^{7}$ photons/bunch, $7.15 \cdot 10^{4} \mathrm{GeV} /$ bunch, mean photon energy $=3.74 \mathrm{MeV}$. CLIC: $7.00 \cdot 10^{5}$ photons/bunch, $1.69 \cdot 10^{3} \mathrm{GeV} /$ bunch, mean photon energy $=2.42 \mathrm{MeV}$. (The normalization of the vertical scale is arbitrary and is different for each machine.) 


\begin{tabular}{|c|c|c|c|}
\hline & TESLA & NLC & CLIC-500 \\
\hline \# bunches /(effective train) & 150 & 192 & 154 \\
\hline \multicolumn{4}{|c|}{ Losses upstream of FD } \\
\hline Mean photon energy $(\mathrm{MeV})$ & 0.450 & 0.032 & 0.034 \\
\hline \# photons/bunch & $1.38 \cdot 10^{10}$ & $0.93 \cdot 10^{9}$ & $5.93 \cdot 10^{8}$ \\
\hline /eff. train & $2.07 \cdot 10^{12}$ & $1.79 \cdot 10^{11}$ & $9.13 \cdot 10^{10}$ \\
\hline \multicolumn{4}{|l|}{ Total photon energy $(\mathrm{GeV})$} \\
\hline /bunch & $6.21 \cdot 10^{6}$ & $2.96 \cdot 10^{4}$ & $2.03 \cdot 10^{4}$ \\
\hline /eff. train & $9.32 \cdot 10^{8}$ & $5.68 \cdot 10^{6}$ & $3.13 \cdot 10^{6}$ \\
\hline \multicolumn{4}{|c|}{ Losses downstream of outgoing FD } \\
\hline Mean photon energy $(\mathrm{MeV})$ & 0.467 & - & - \\
\hline \# photons/bunch & $4.75 \cdot 10^{8}$ & - & - \\
\hline /eff. train & $7.14 \cdot 10^{10}$ & - & - \\
\hline Total photon energy $(\mathrm{GeV})$ & & & \\
\hline /bunch & $2.22 \cdot 10^{5}$ & - & - \\
\hline /eff. train & $3.33 \cdot 10^{7}$ & - & - \\
\hline
\end{tabular}

Table 10: Synchrotron radiation from the beam core hitting IR SR masks. The photon energy spectrum is displayed in Figure 13. The losses tabulated refer to masks DUMP1 for TESLA and DUMP2 for NLC and CLIC of the corresponding beam line. The number of bunches per 'effective' train reflects the sensitivity window of the TPC. It is equal to 150 bunches $(50 \mu \mathrm{s})$ for TESLA, and to the nominal number of bunches per train for NLC and CLIC.

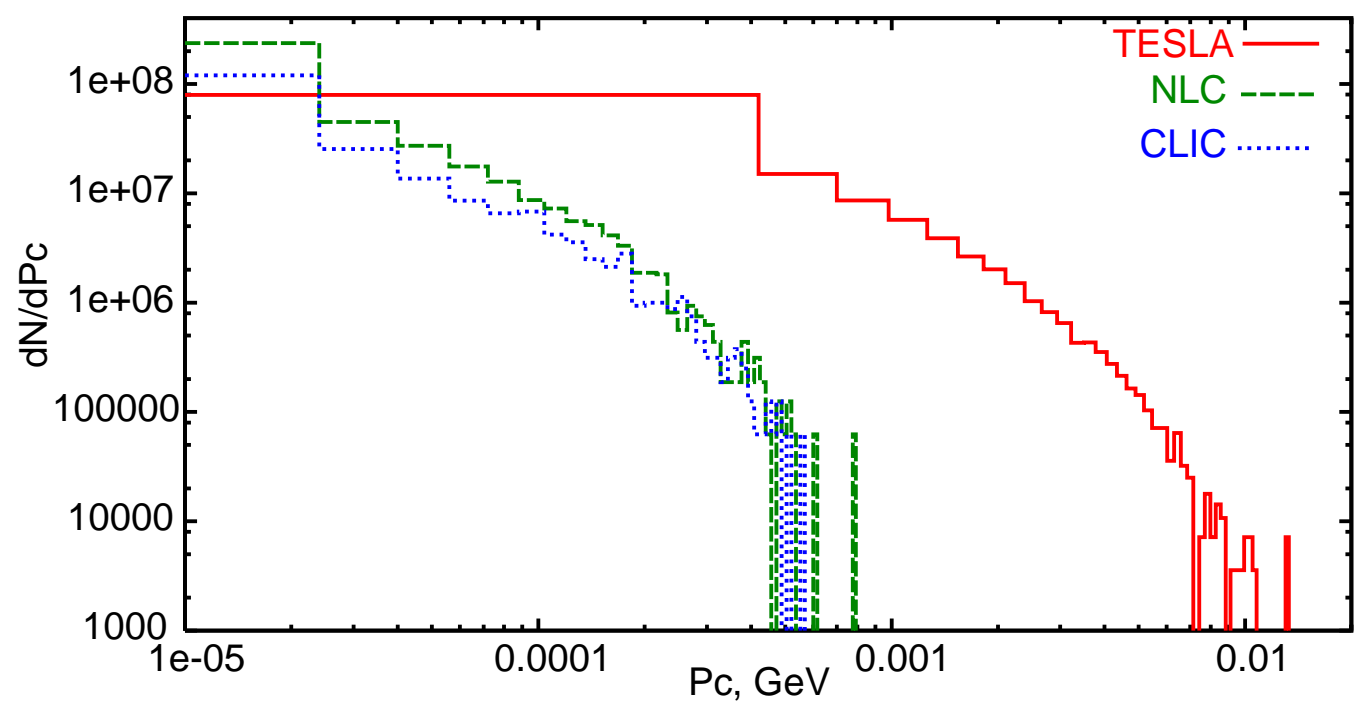

Figure 13: Energy spectrum of synchrotron-radiation photons from the beam core, that hit DUMP1 (for TESLA) or DUMP2 (for NLC and CLIC). (The normalization of the vertical scale is arbitrary and is different for each machine.) 


\section{Appendix: Loss Patterns and Halo Characterization}
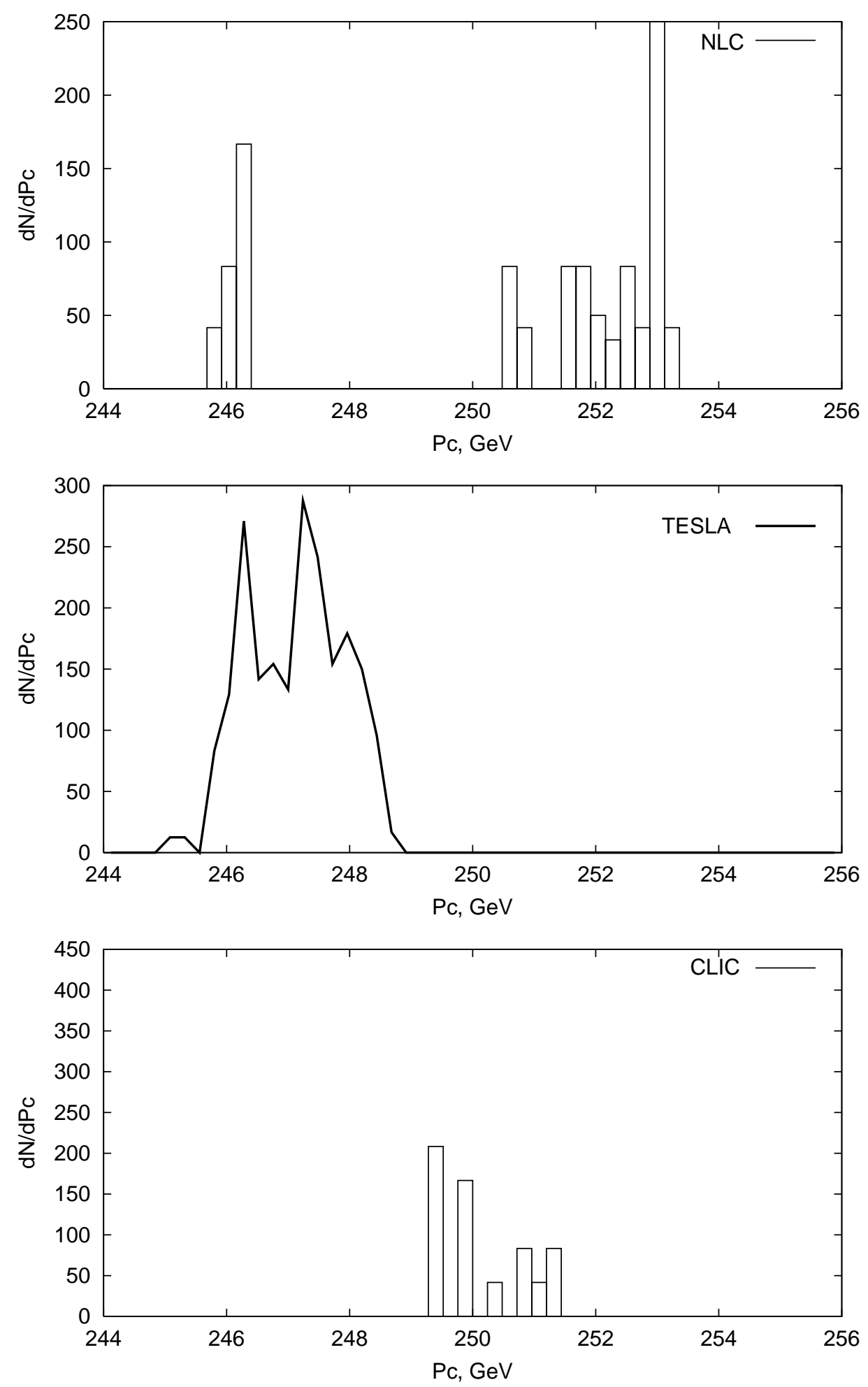

Figure 14: Energy spectrum of primary particles falling outside the effective collimation depth (as defined in Table 7) at the FD entrance, with the collimation settings listed in Tables 2-4, for NLC (top), TESLA (middle) and CLIC (bottom). The incoming halo was represented by $10^{5}$ rays incident into the BDS. 

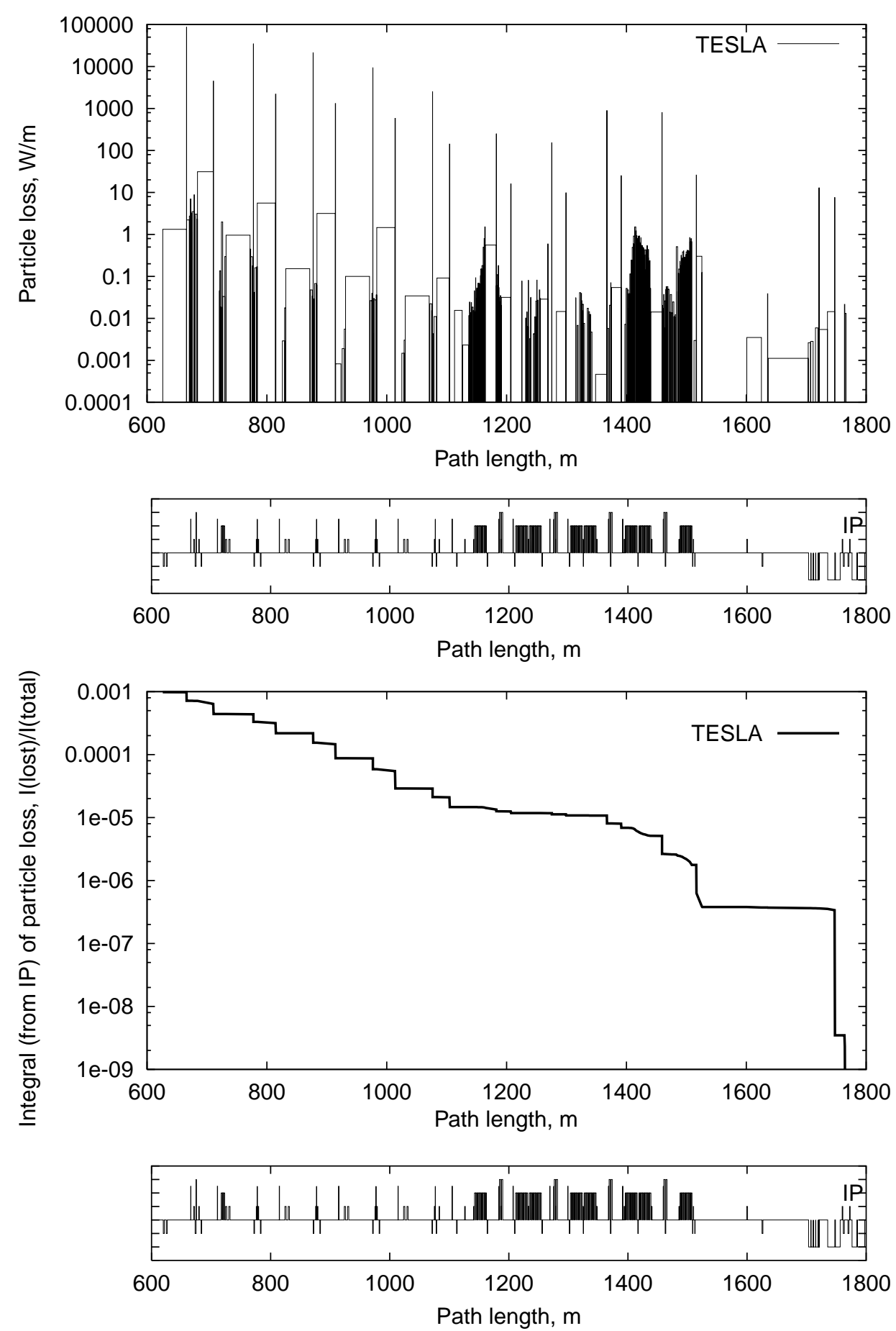

Figure 15: TESLA halo particle loss distributions (top) and loss integral (bottom). 

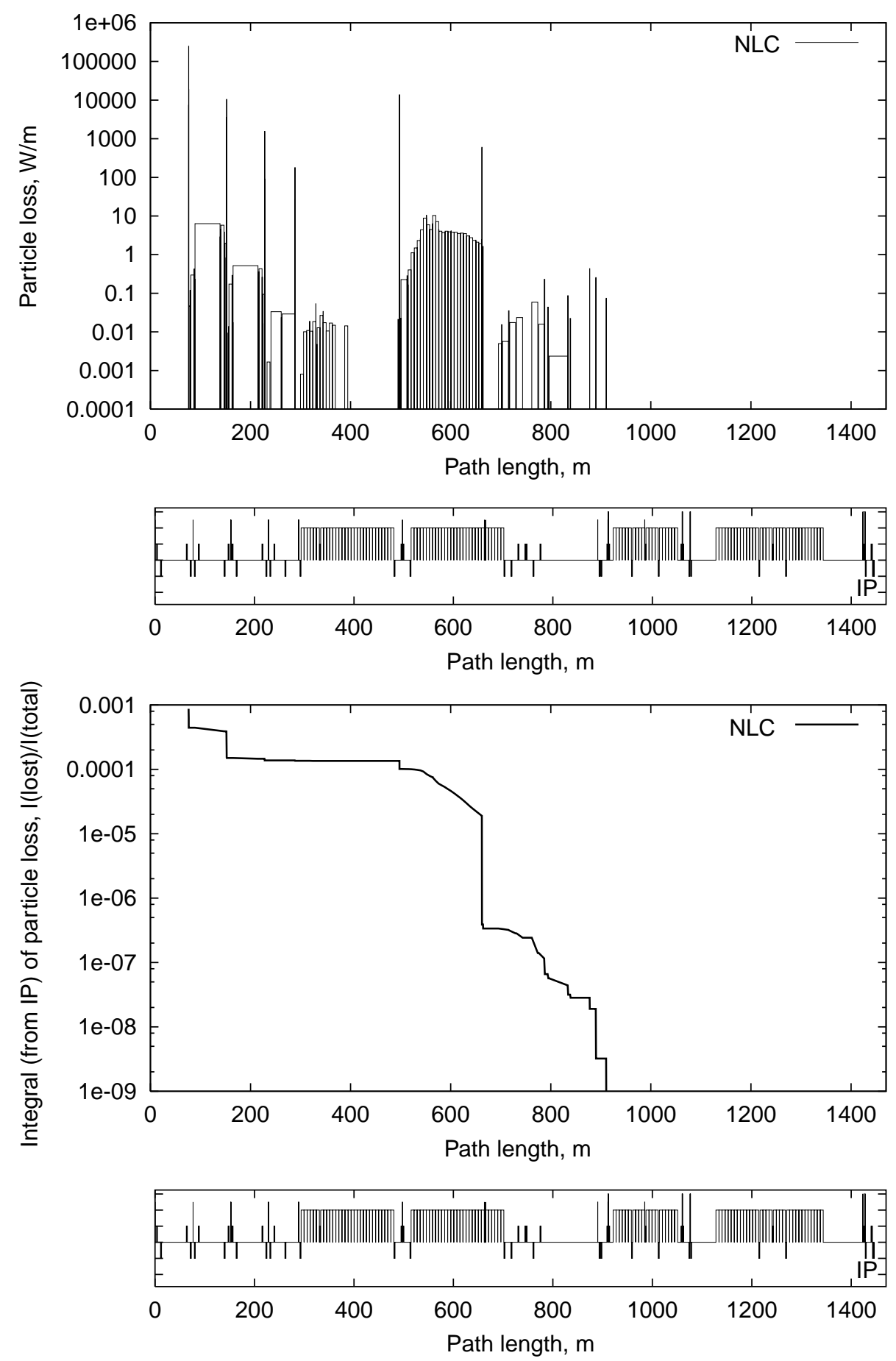

Figure 16: NLC halo particle loss distributions (top) and loss integral(bottom). 

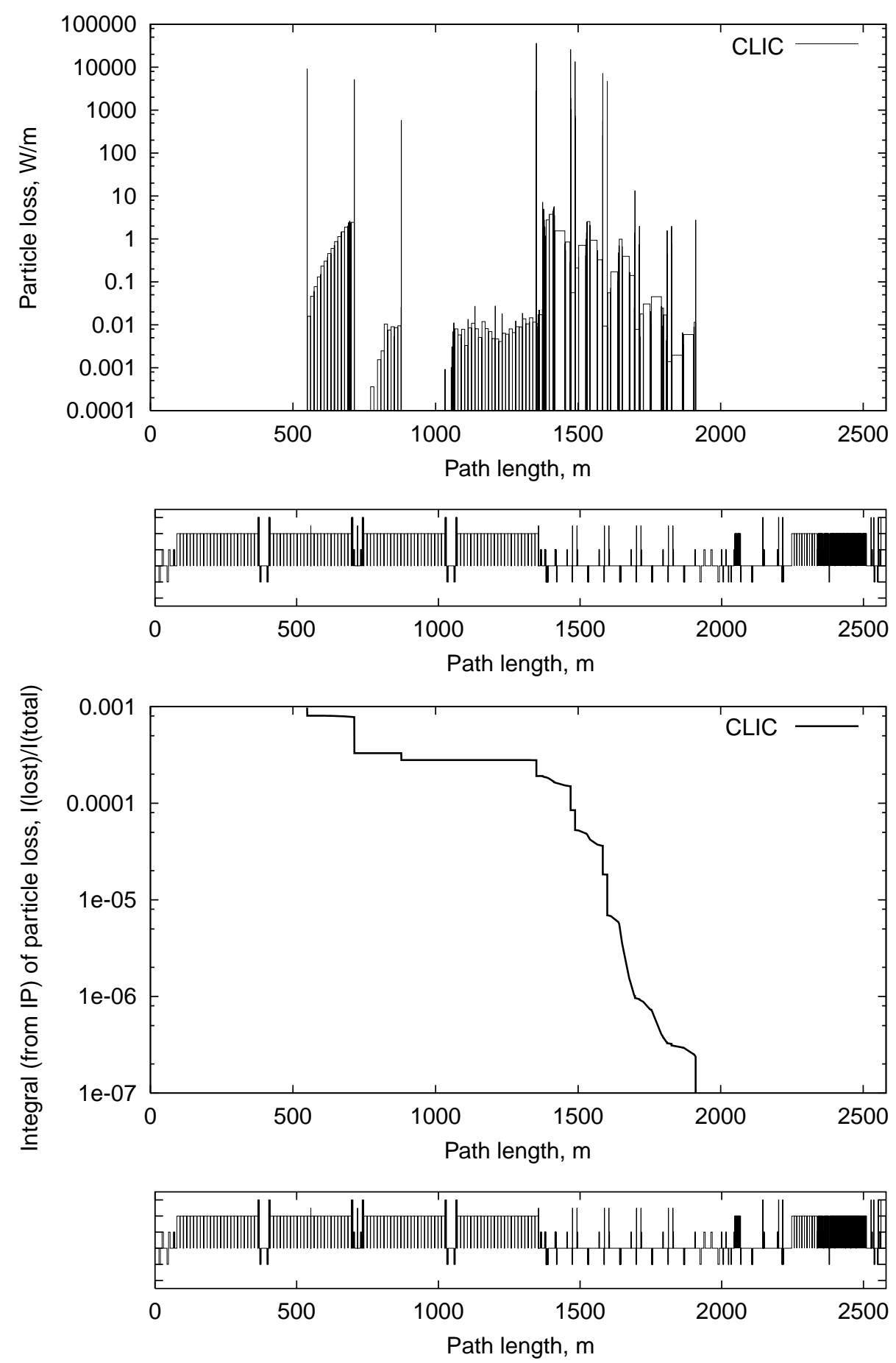

Figure 17: CLIC halo particle loss distributions (top) and loss integral (bottom). 

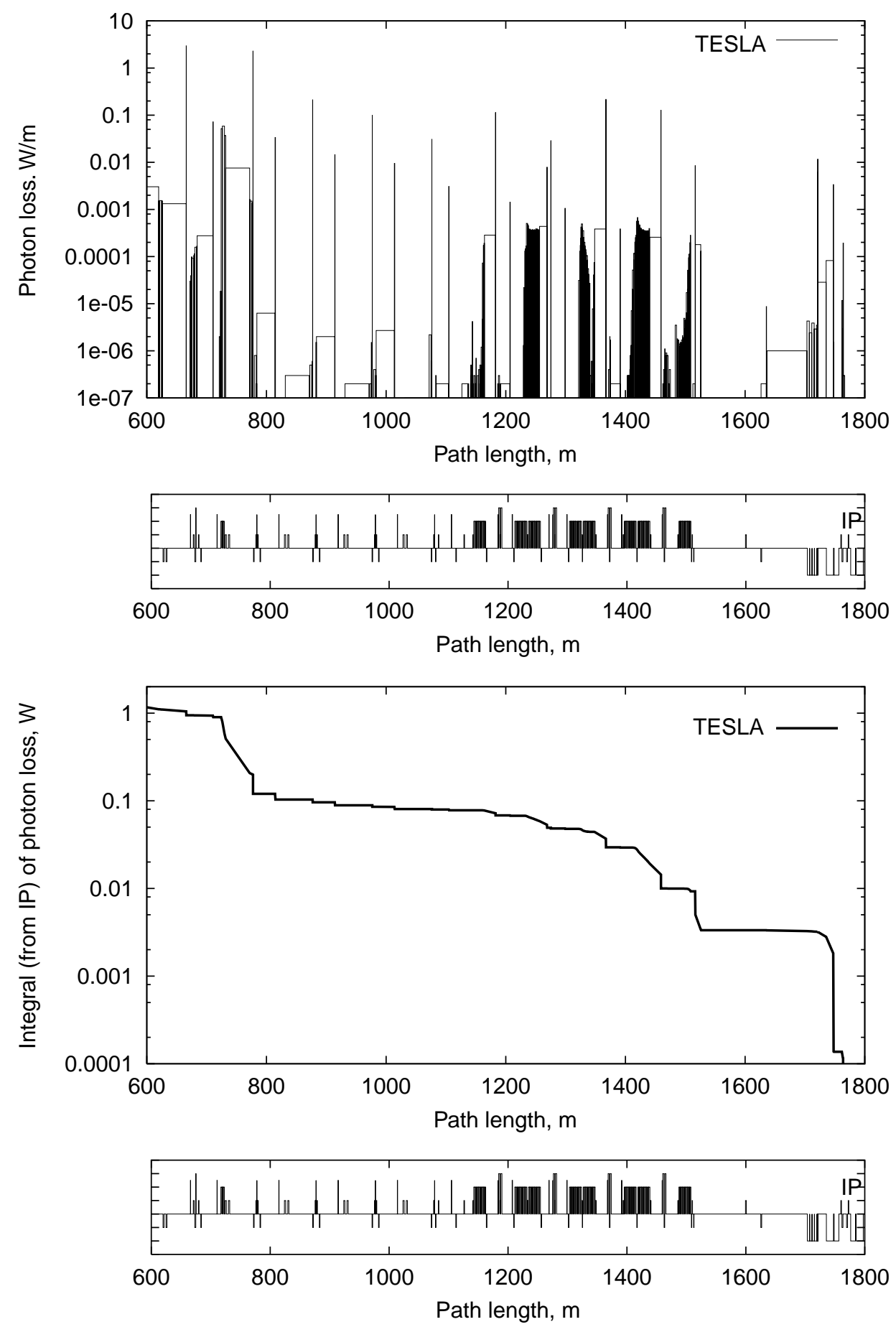

Figure 18: Synchrotron radiation losses from the beam halo in TESLA: longitudinal loss distribution (top), integrated losses starting back from the IP (bottom). 

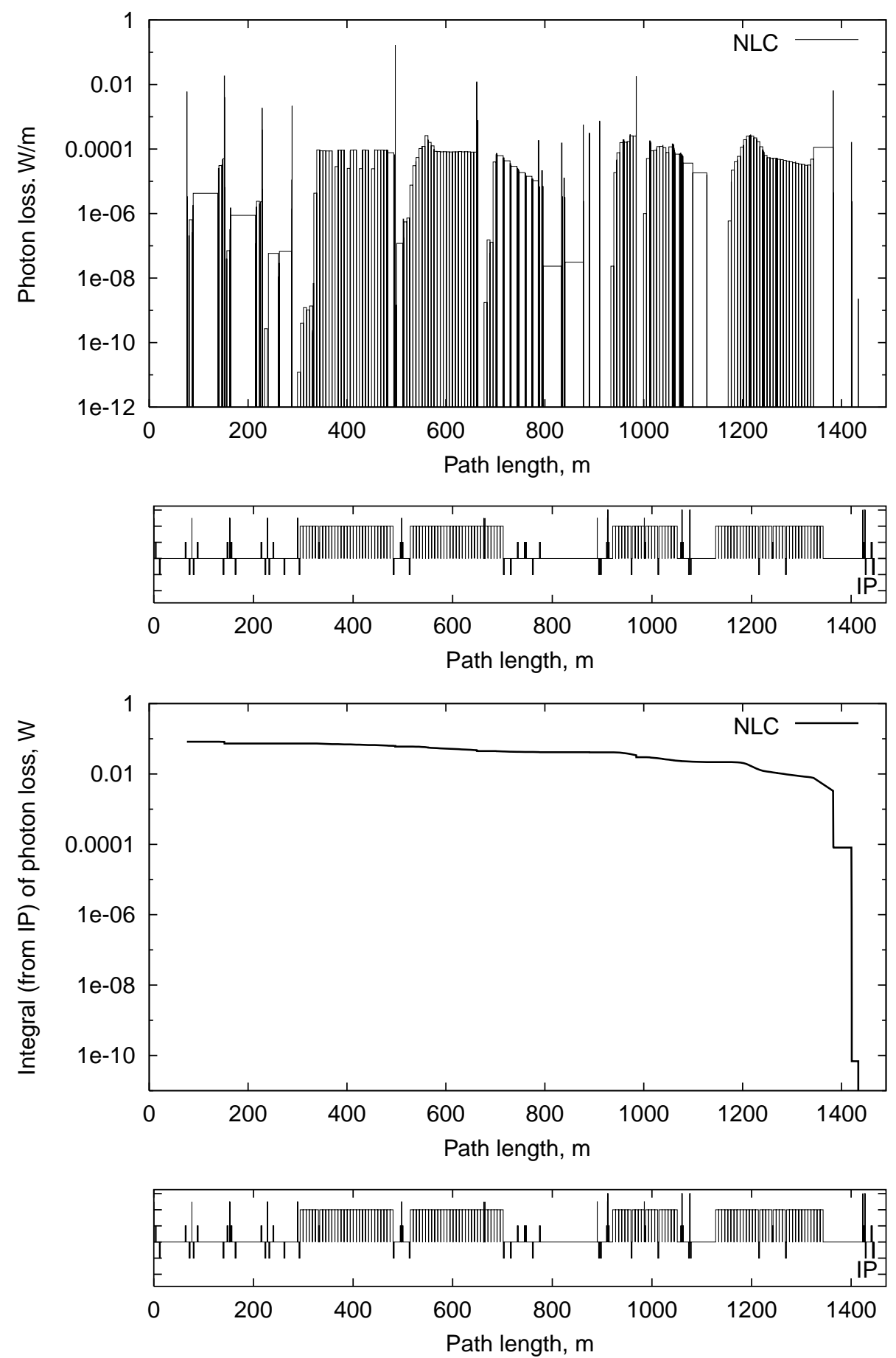

Figure 19: Synchrotron radiation losses from the beam halo in NLC: longitudinal loss distribution (top), integrated losses starting back from the IP (bottom). 

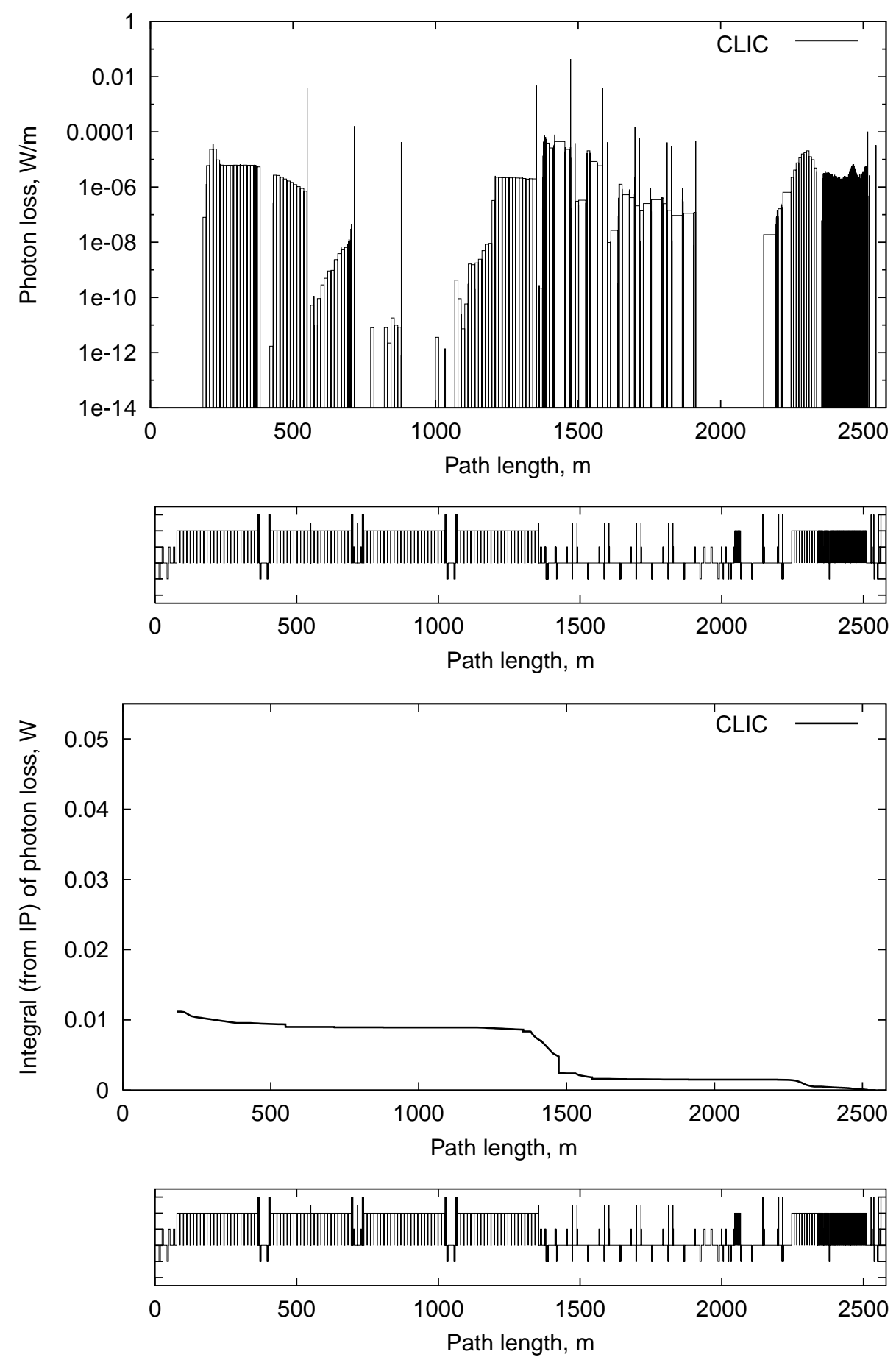

Figure 20: Synchrotron radiation losses from the beam halo in CLIC: longitudinal loss distribution (top), integrated losses starting back from the IP (bottom). 

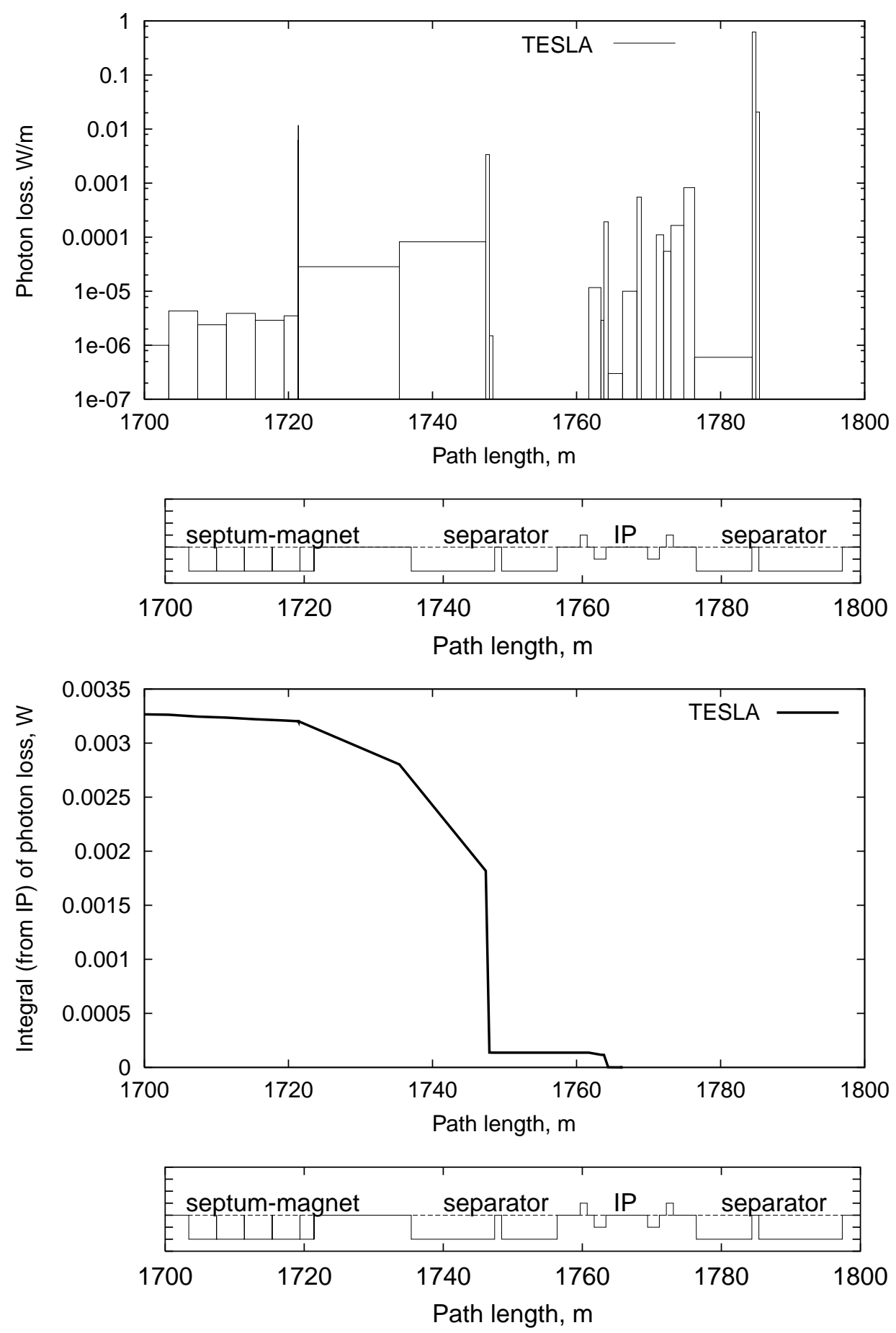

Figure 21: Synchrotron radiation losses from the beam halo near the TESLA IP: longitudinal loss distribution (top), integrated losses starting back from the IP (bottom). The IP is at $1766.393 \mathrm{~m}$. 

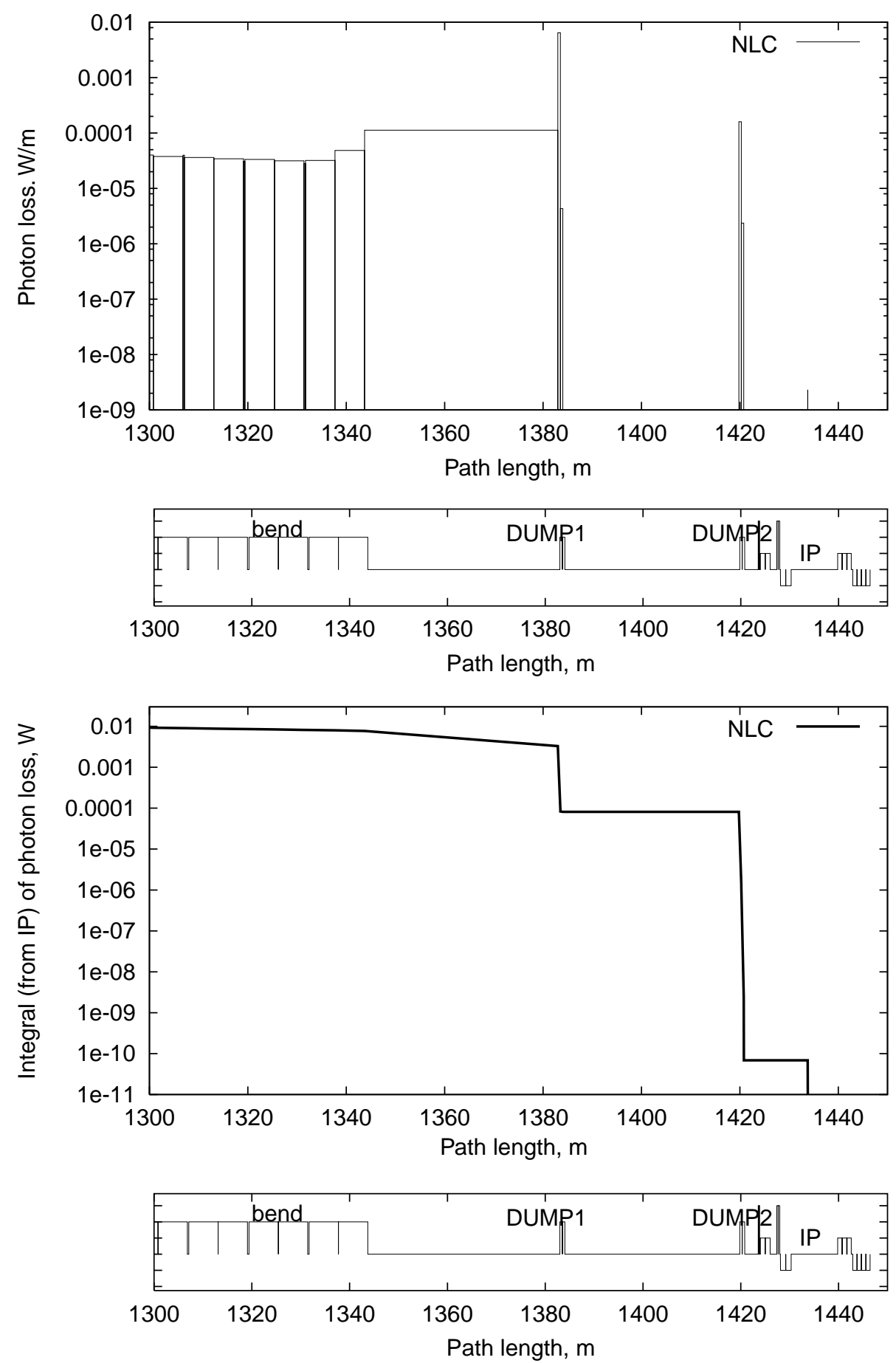

Figure 22: Synchrotron radiation losses from the beam halo near the NLC IP: longitudinal loss distribution (top), integrated losses starting back from the IP (bottom). The IP is at $1433.815 \mathrm{~m}$. 

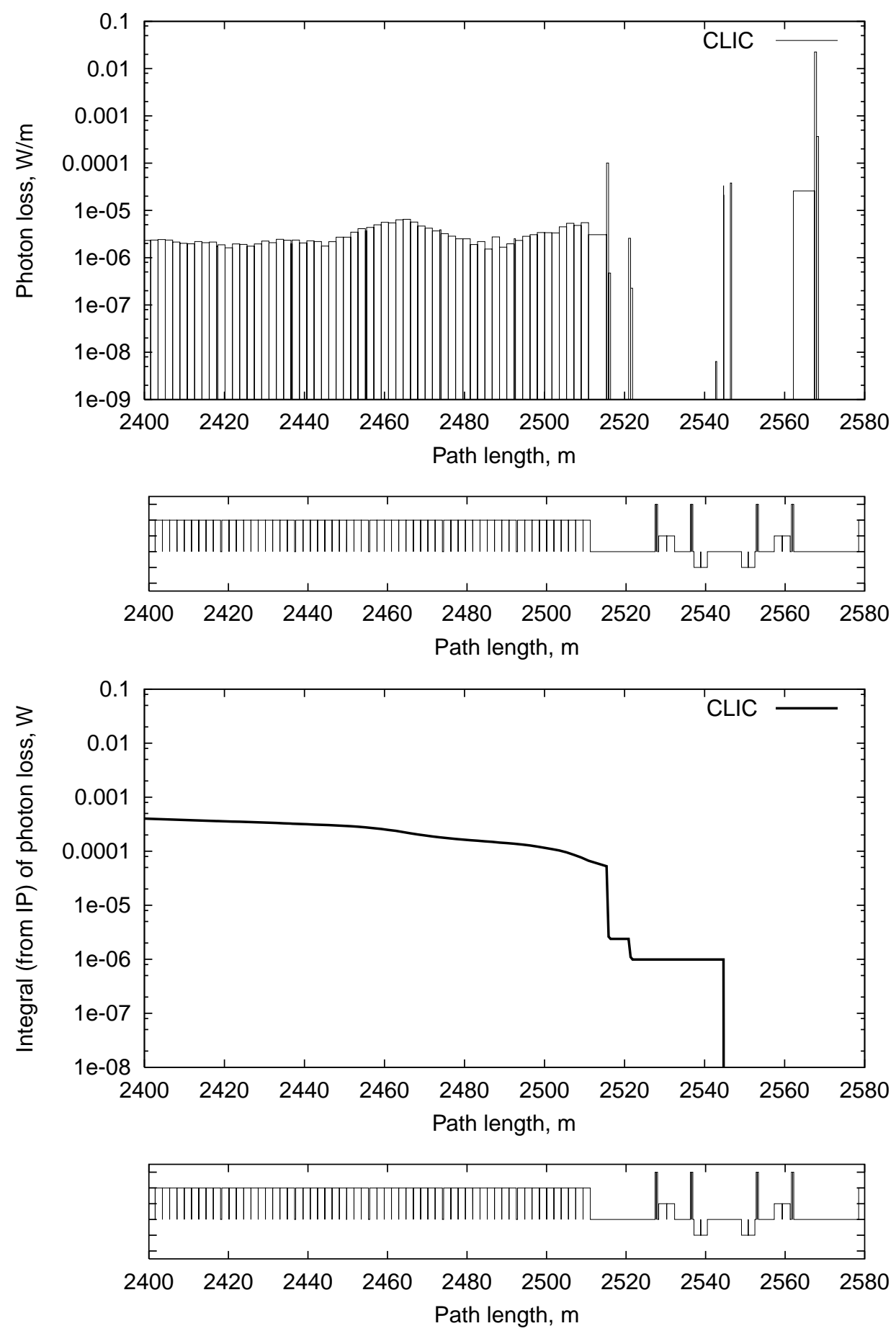

Figure 23: Synchrotron radiation losses from the beam halo near the CLIC IP: longitudinal loss distribution (top), integrated losses starting back from the IP (bottom). The IP is at $2544.74 \mathrm{~m}$. 

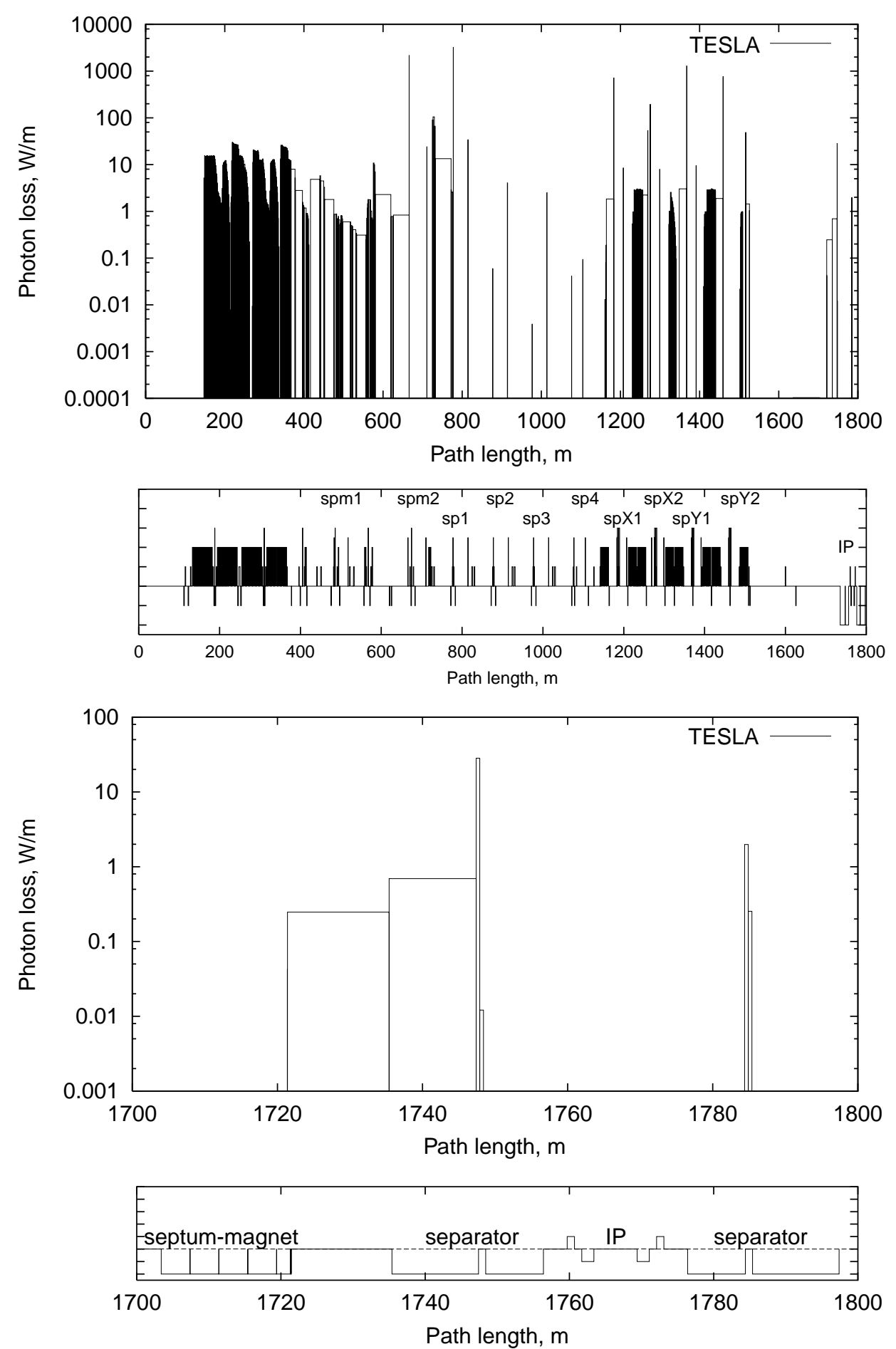

Figure 24: TESLA synchrotron radiation loss distributions from the beam core (top) and near the IP (bottom). The IP is at $1766.393 \mathrm{~m}$. The photon loss in DUMP1 is $14.5 \mathrm{~W}$. 

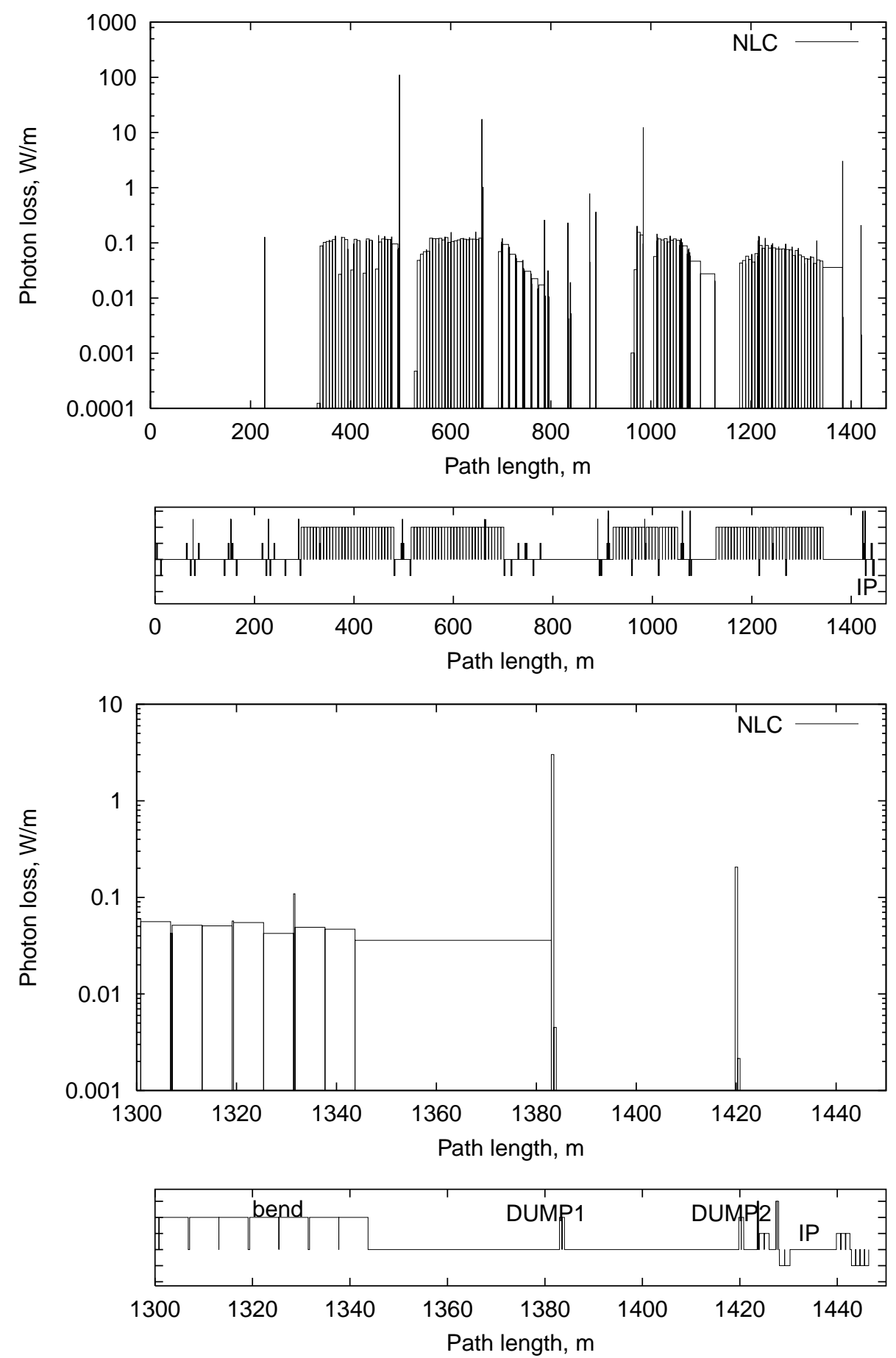

Figure 25: NLC synchrotron radiation loss distributions from the beam core (top) and near the IP (bottom). The IP is at $1433.815 \mathrm{~m}$. The photon loss is $1.52 \mathrm{~W}$ in DUMP1 and $0.11 \mathrm{~W}$ in DUMP2 . 

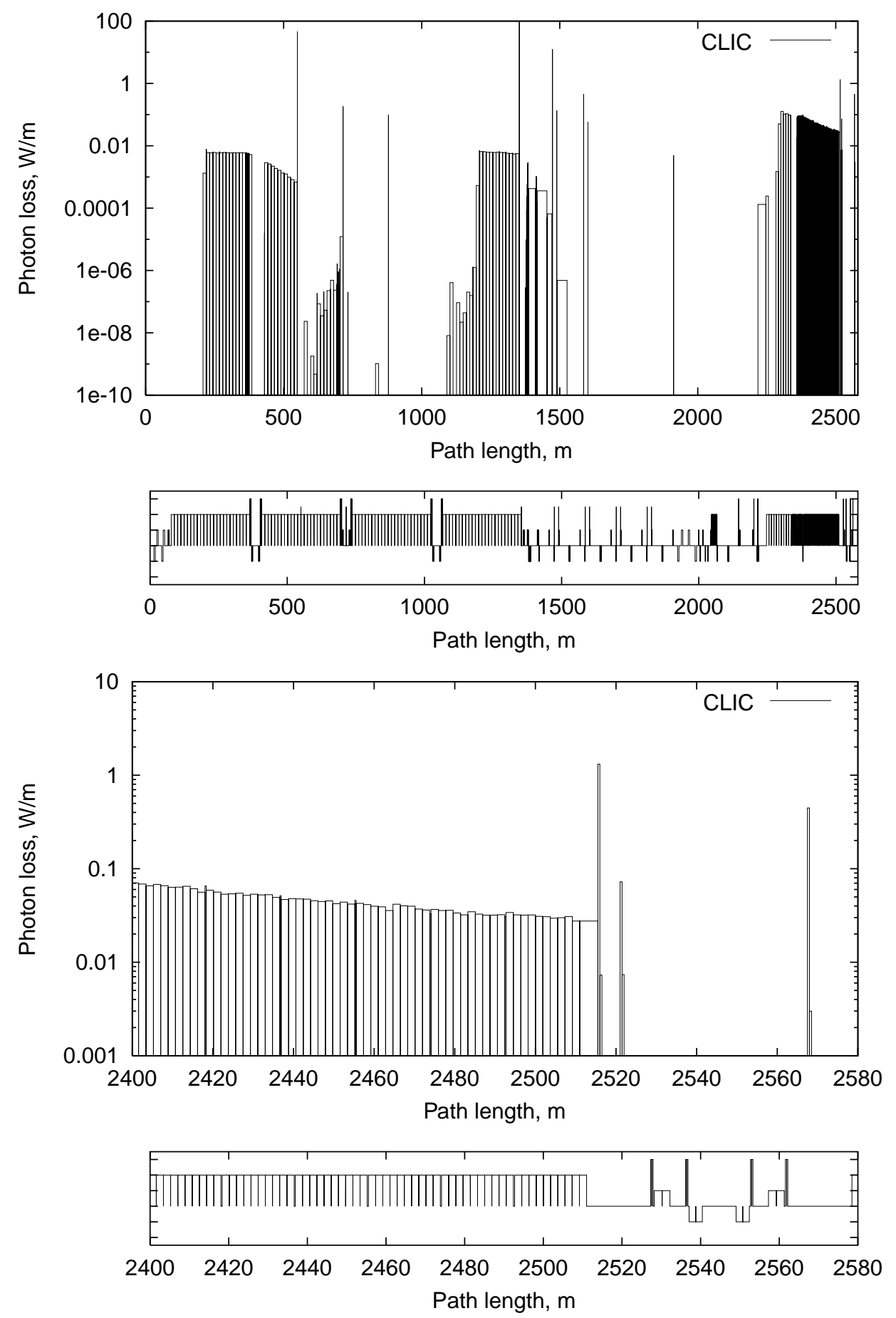

Figure 26: CLIC synchrotron radiation loss distributions from the beam core (top) and near the IP (bottom). The IP is at $2544.74 \mathrm{~m}$. The photon loss is $0.66 \mathrm{~W}$ in DUMP1 and $0.04 \mathrm{~W}$ in DUMP2. 

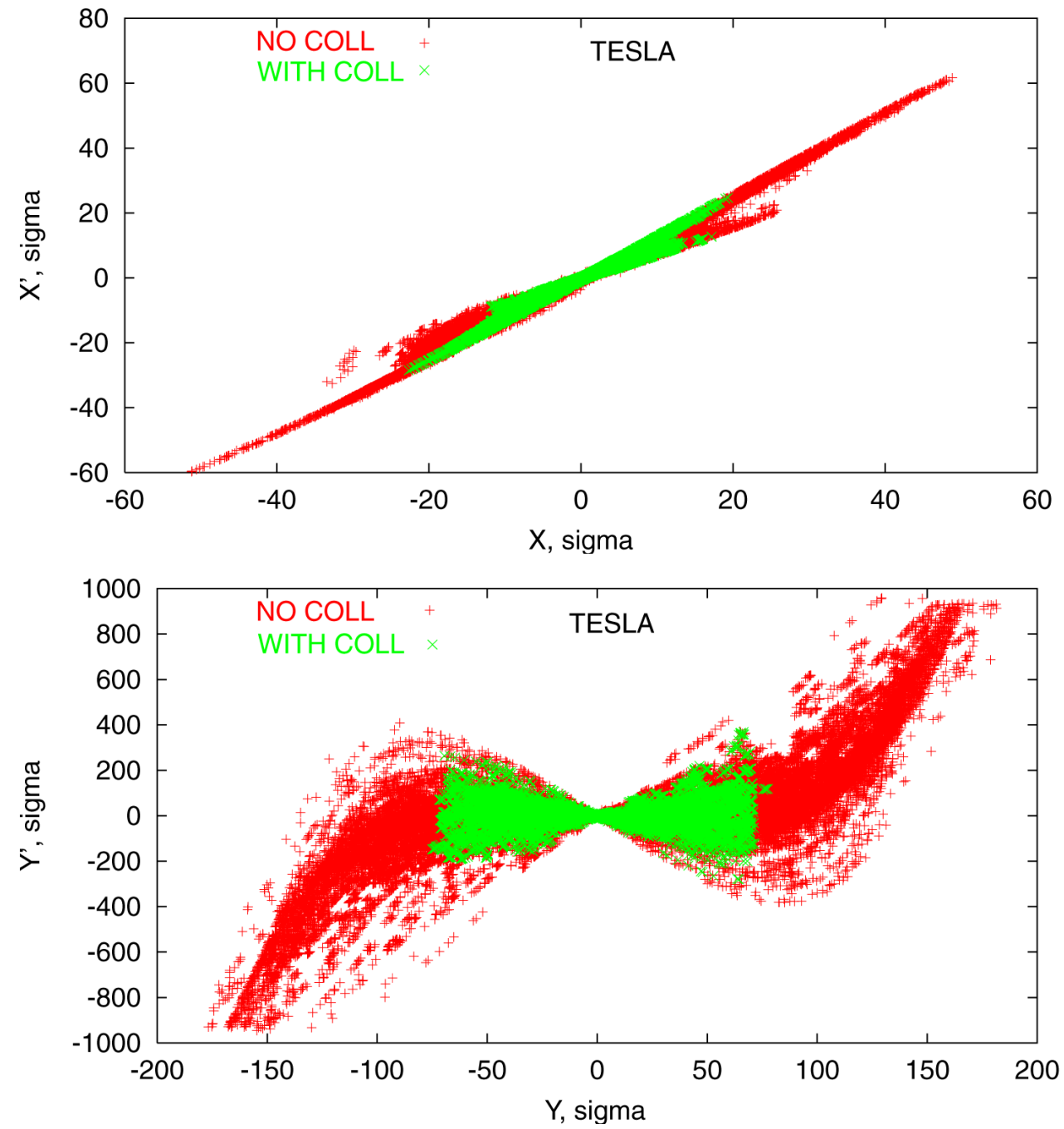

Figure 27: TESLA halo particle population with and without collimation in the phase plane at the FF doublet entrance. 

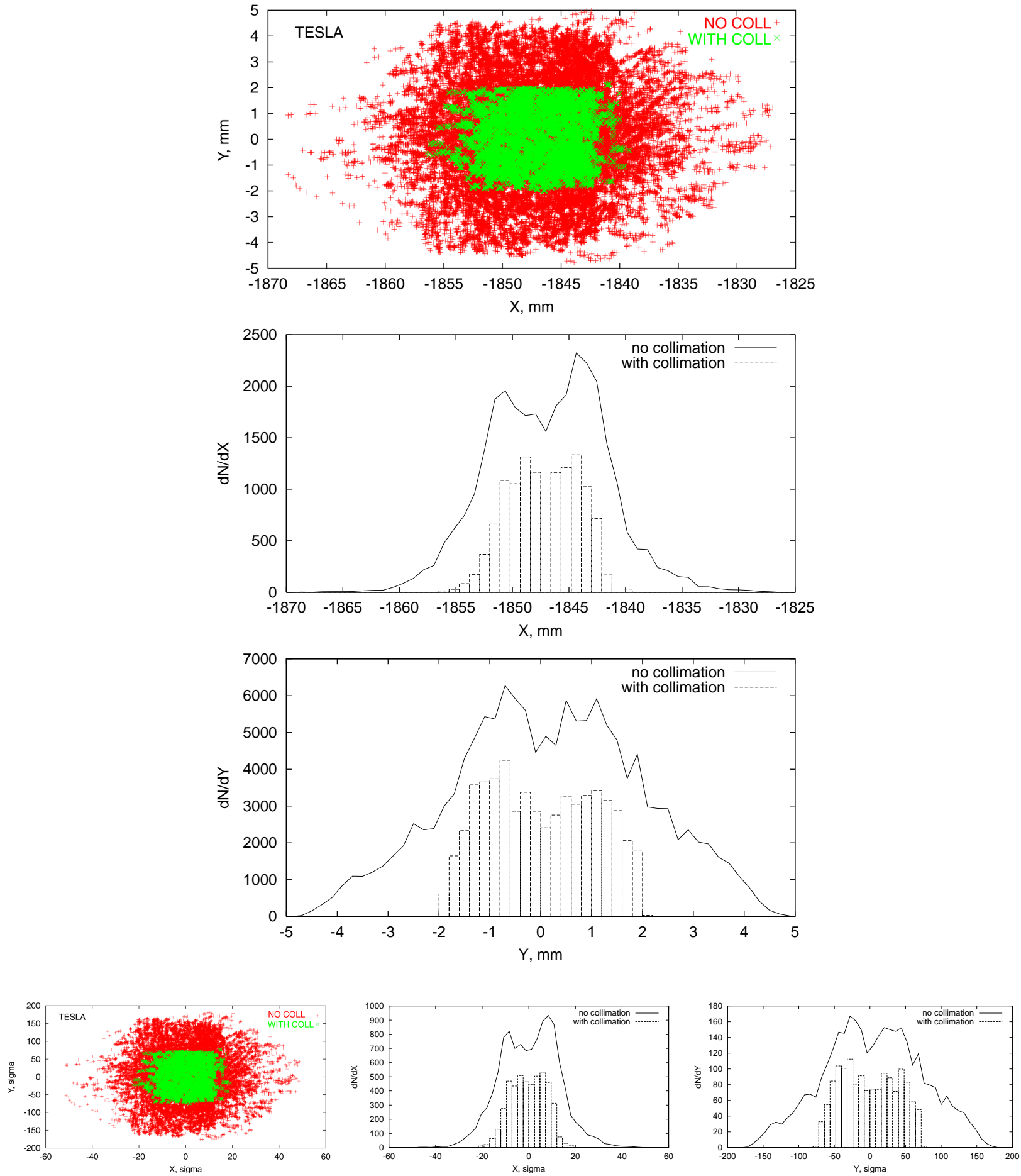

Figure 28: TESLA halo particle population and distributions at the FF doublet entrance. The bottom pictures are the same plots in units of beam sigma. 

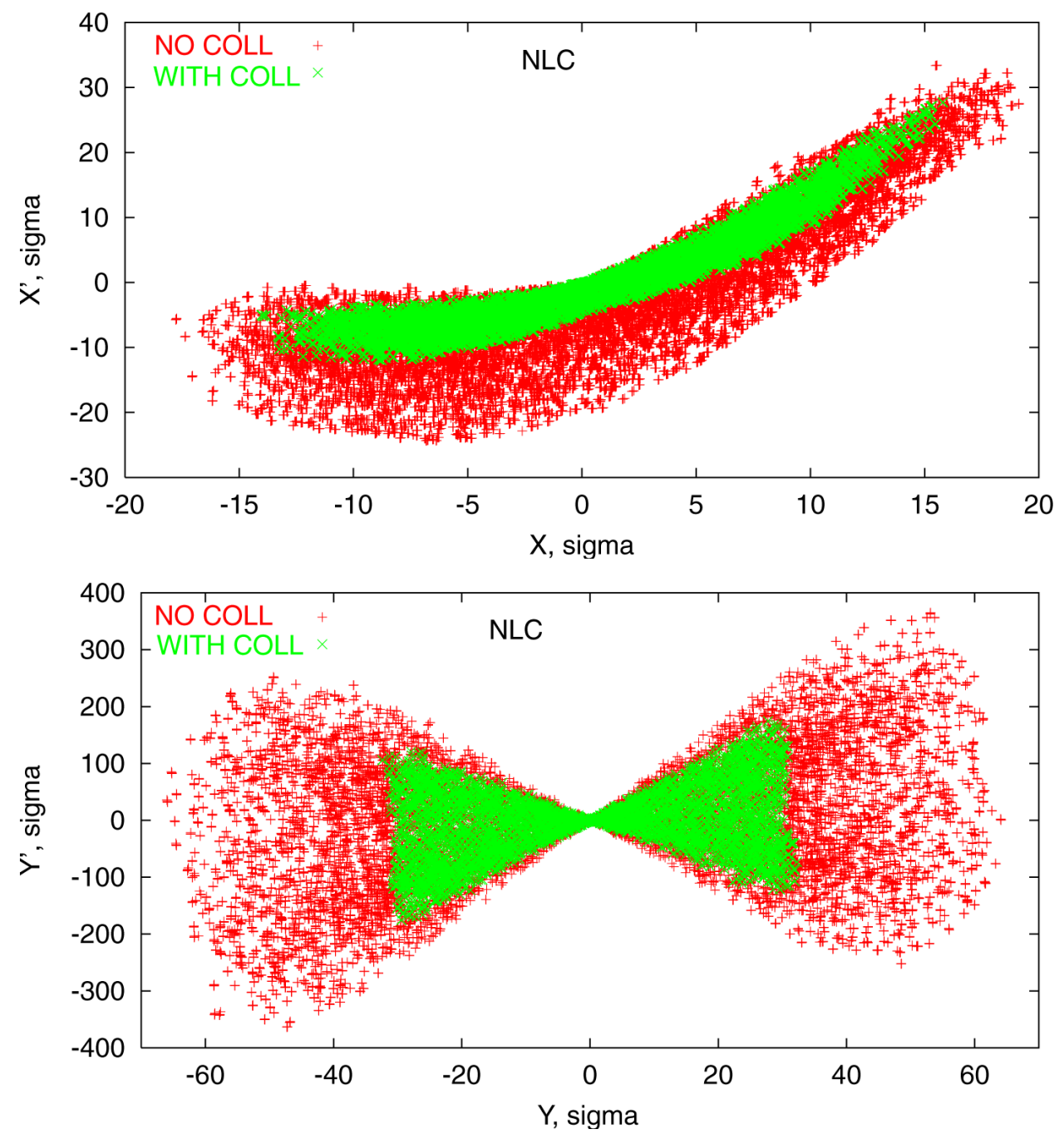

Figure 29: NLC halo particle population with and without collimation in the phase plane at the FF doublet entrance. 

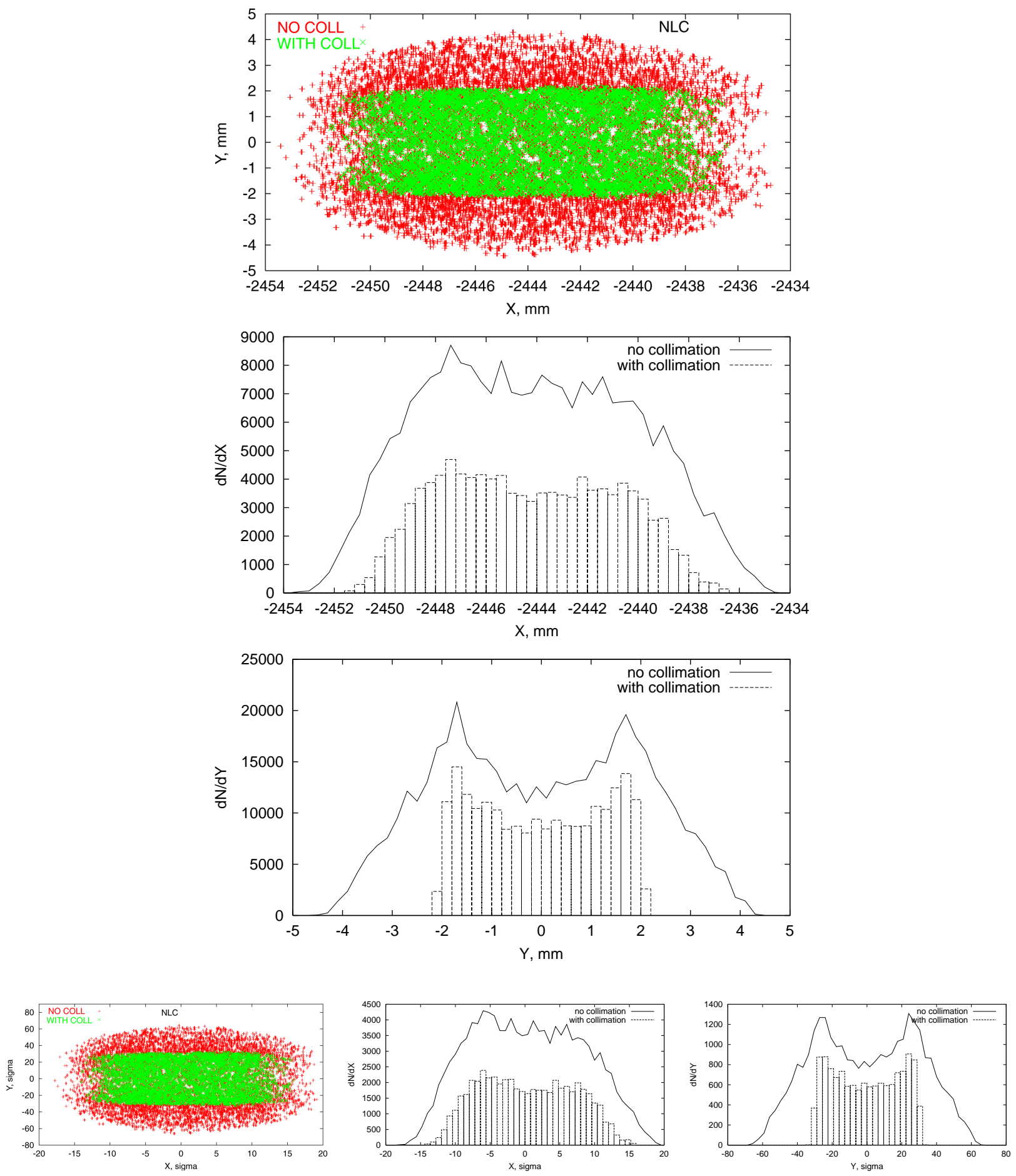

Figure 30: NLC halo particle population and distributions at the FF doublet entrance. The bottom pictures are the same plots in units of beam sigma. 

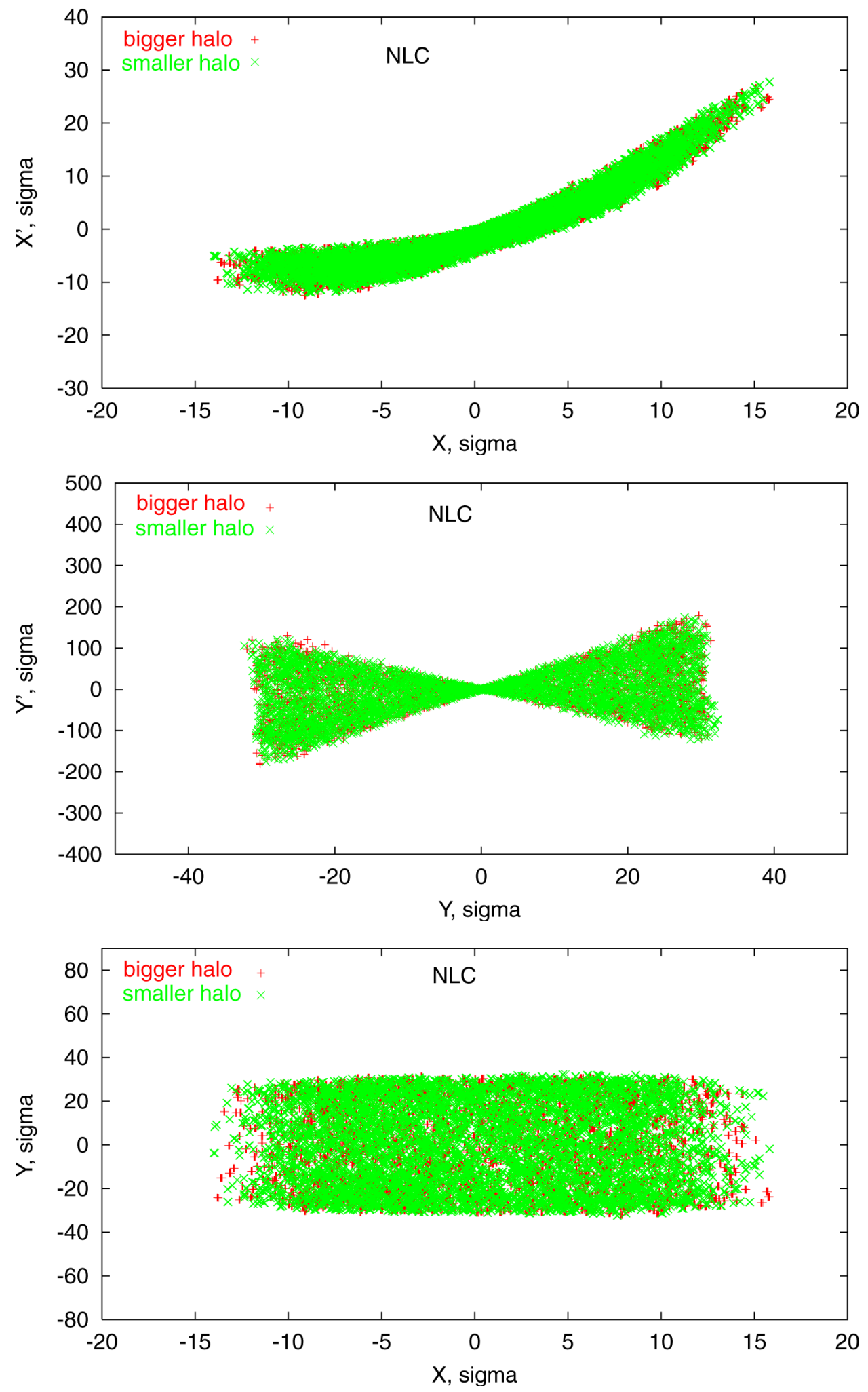

Figure 31: NLC halo particle population with collimation in the phase plane at the FF doublet entrance for the nominal initial halo of $A_{x}=(6-16) \sigma_{x}$ and $A_{y}=(24-73) \sigma_{y}$ (green), and for a larger halo with $A_{x}=(6-24) \sigma_{x}$ and $A_{y}=(24-100) \sigma_{y}$ (red). 

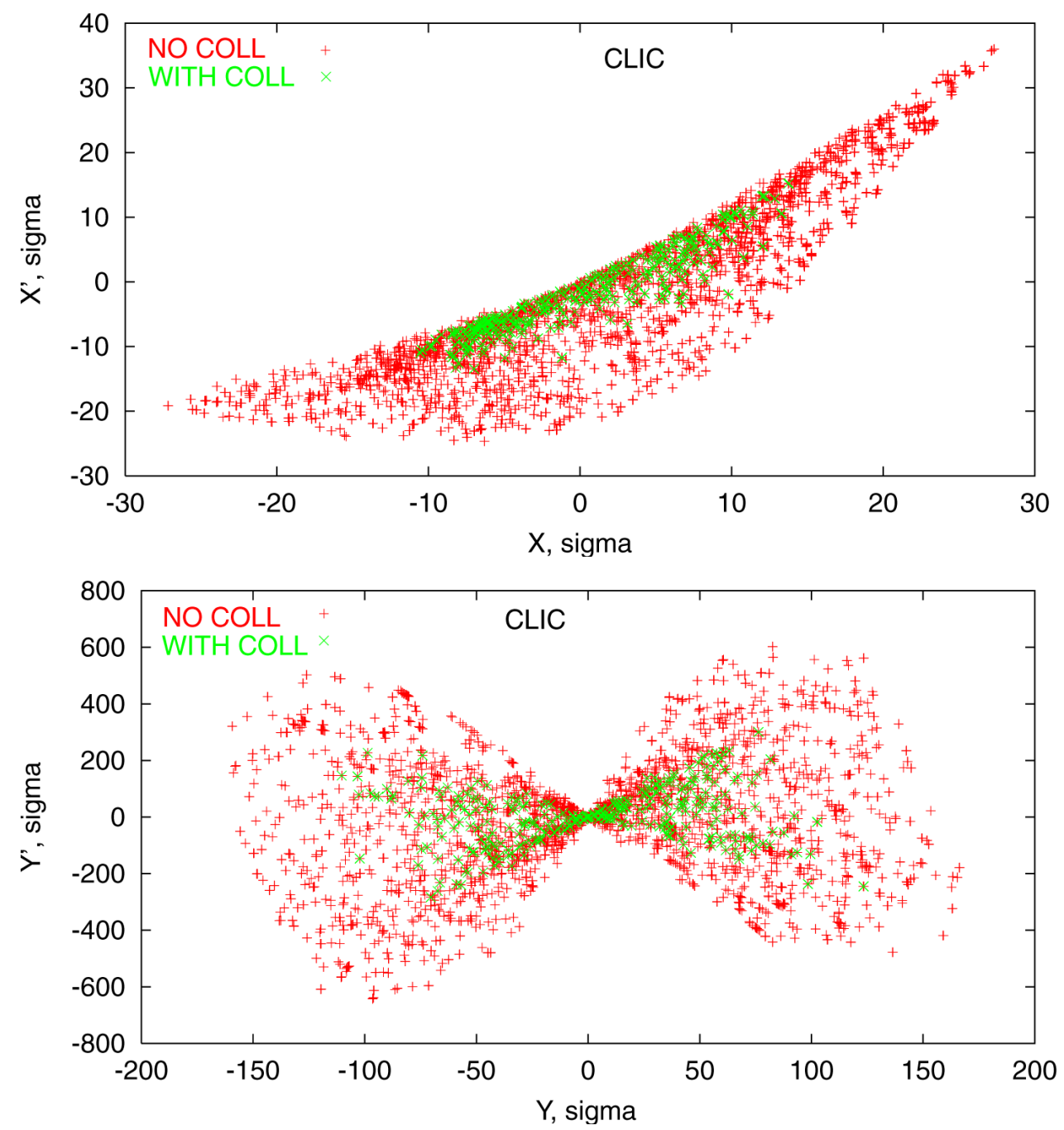

Figure 32: CLIC halo particle population with and without collimation in the phase plane at the FF doublet entrance. 

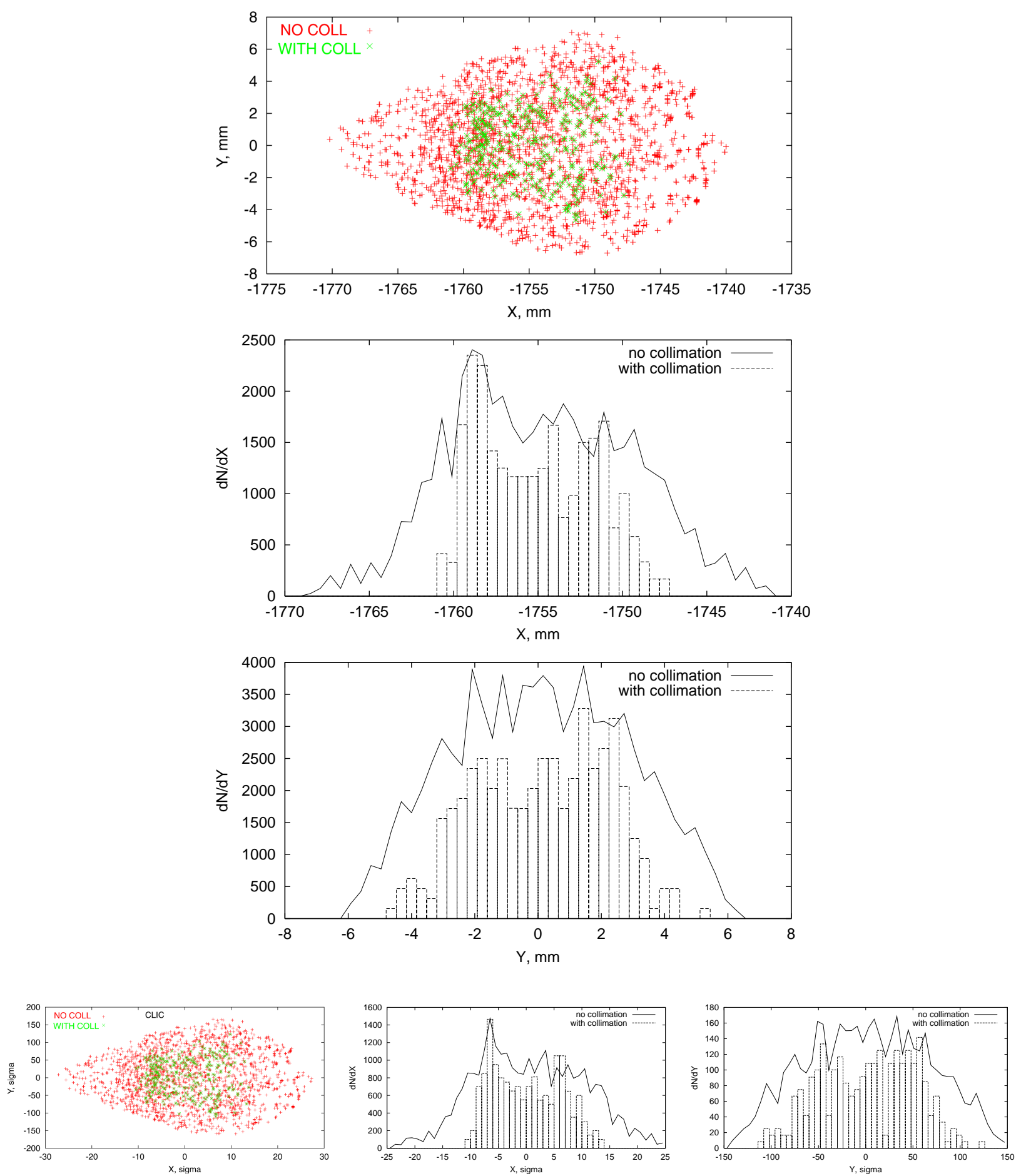

Figure 33: CLIC halo particle population and distributions at the FF doublet entrance. The bottom pictures are the same plots in units of beam sigma. 

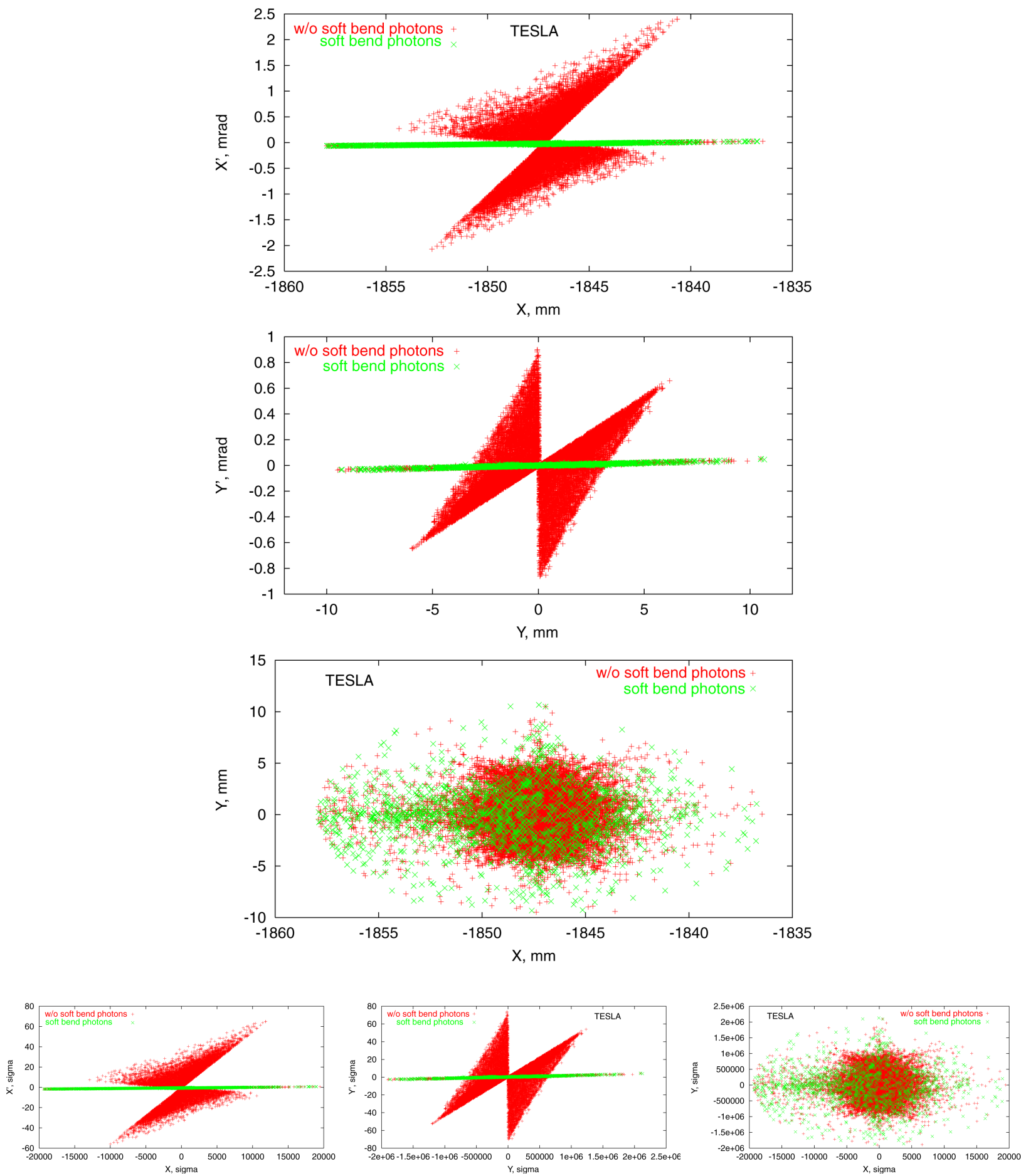

Figure 34: TESLA synchrotron radiation population from beam halo in the phase plane at the IP. Green (grey) are photons from the soft bend. The bottom pictures are the same plots in units of beam sigma. 

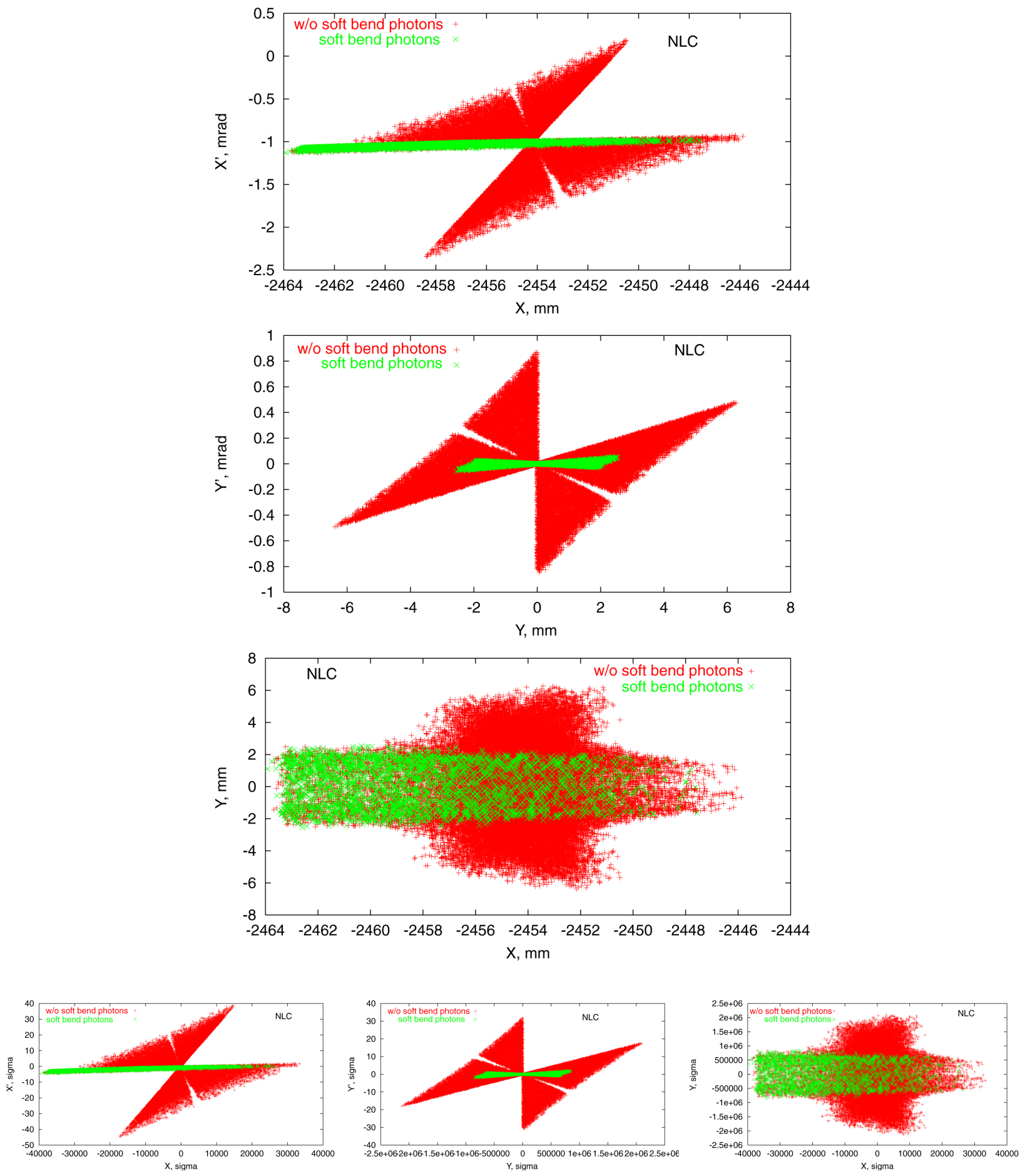

Figure 35: NLC synchrotron radiation population from beam halo in the phase plane at the IP. Green (grey) are photons from the soft bend. The bottom pictures are the same plots in units of beam sigma. 

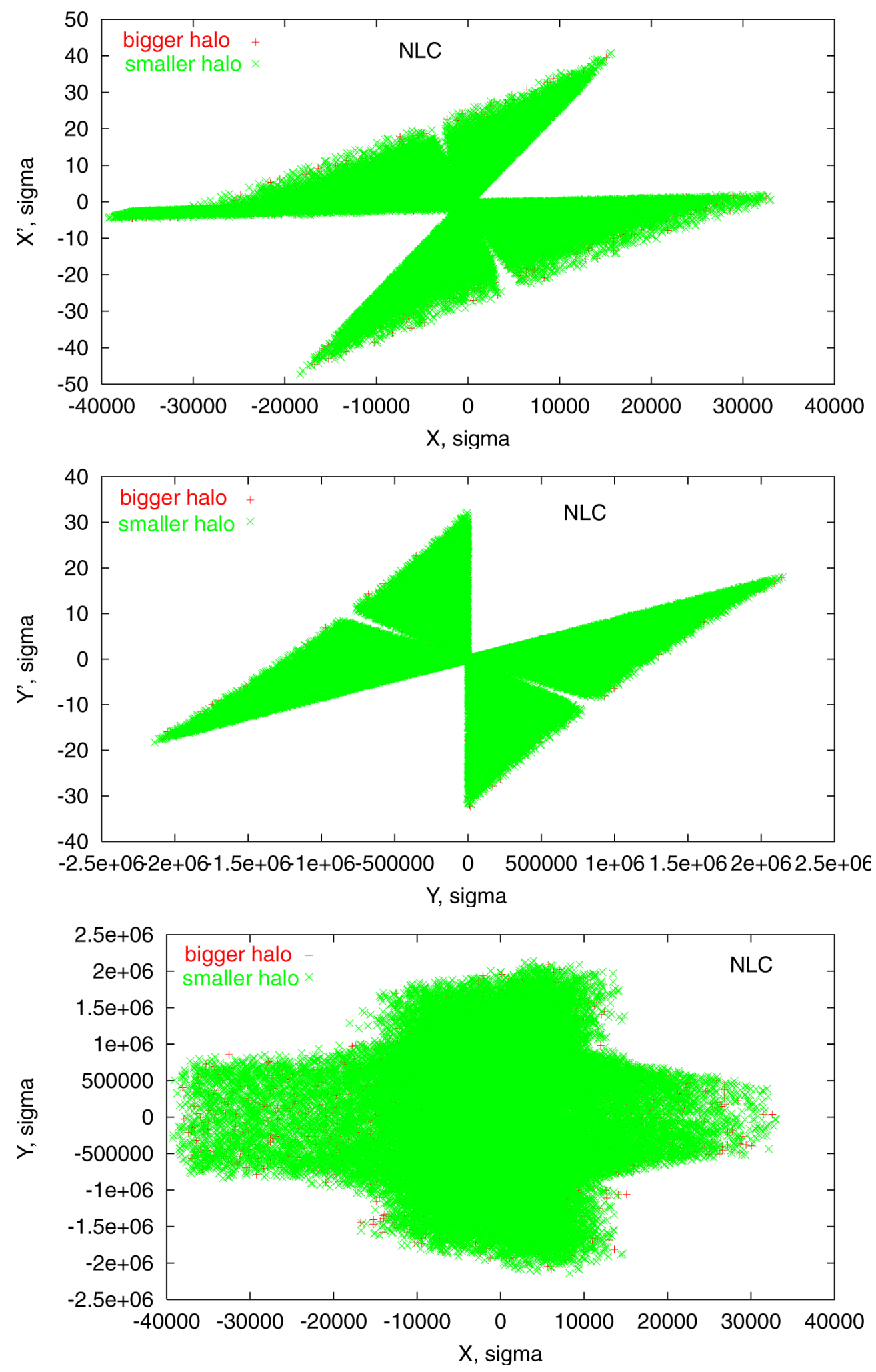

Figure 36: NLC synchrotron radiation population with collimation in the phase plane at the FF doublet entrance for the nominal initial halo of $A_{x}=(6-16) \sigma_{x}$ and $A_{y}=(24-73) \sigma_{y}$ (green), and for a larger halo with $A_{x}=(6-24) \sigma_{x}$ and $A_{y}=(24-100) \sigma_{y}$ (red). 

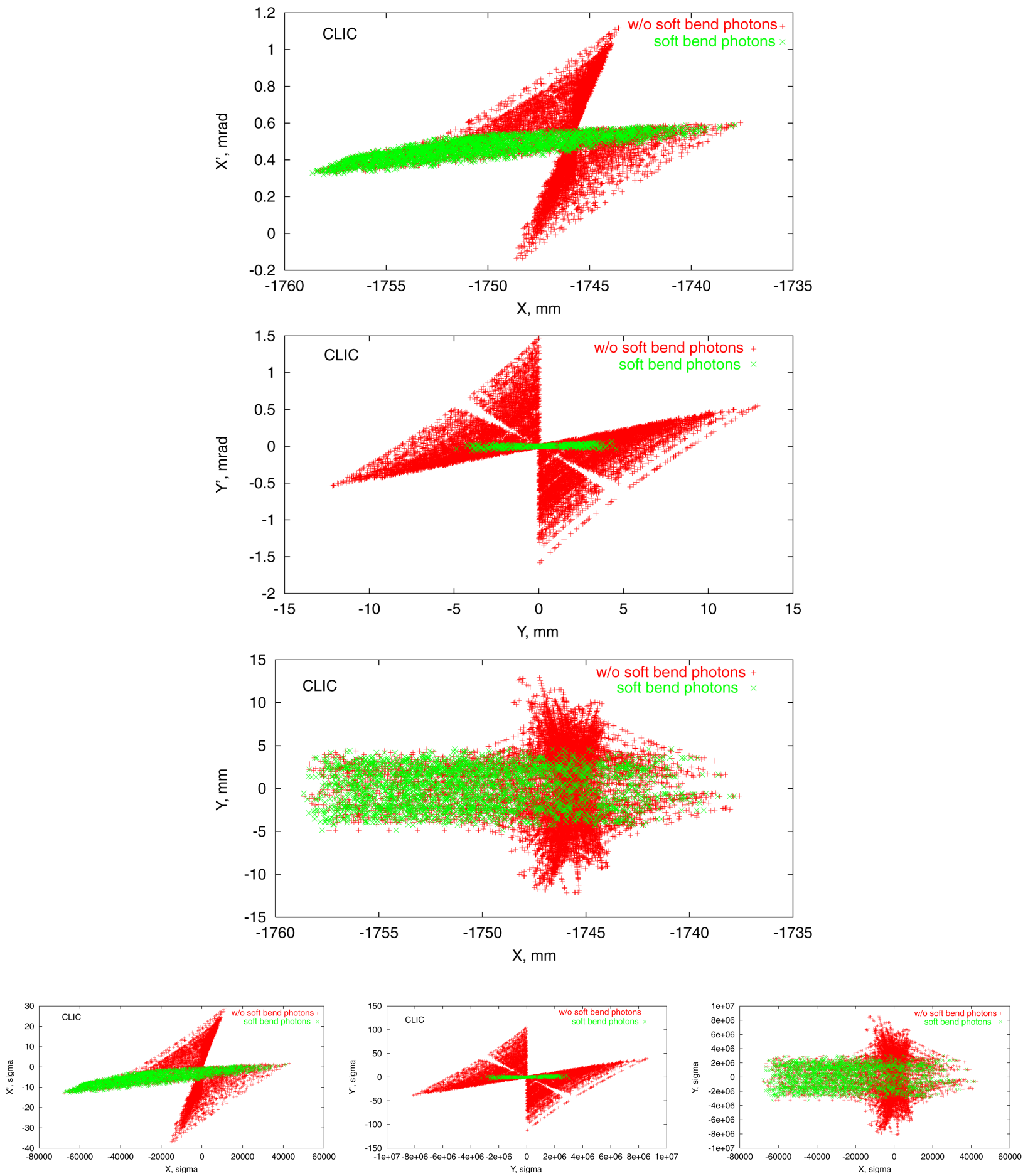

Figure 37: CLIC synchrotron radiation population from beam halo in the phase plane at the IP. Green (grey) are photons from the soft bend. The bottom pictures are the same plots in units of beam sigma. 

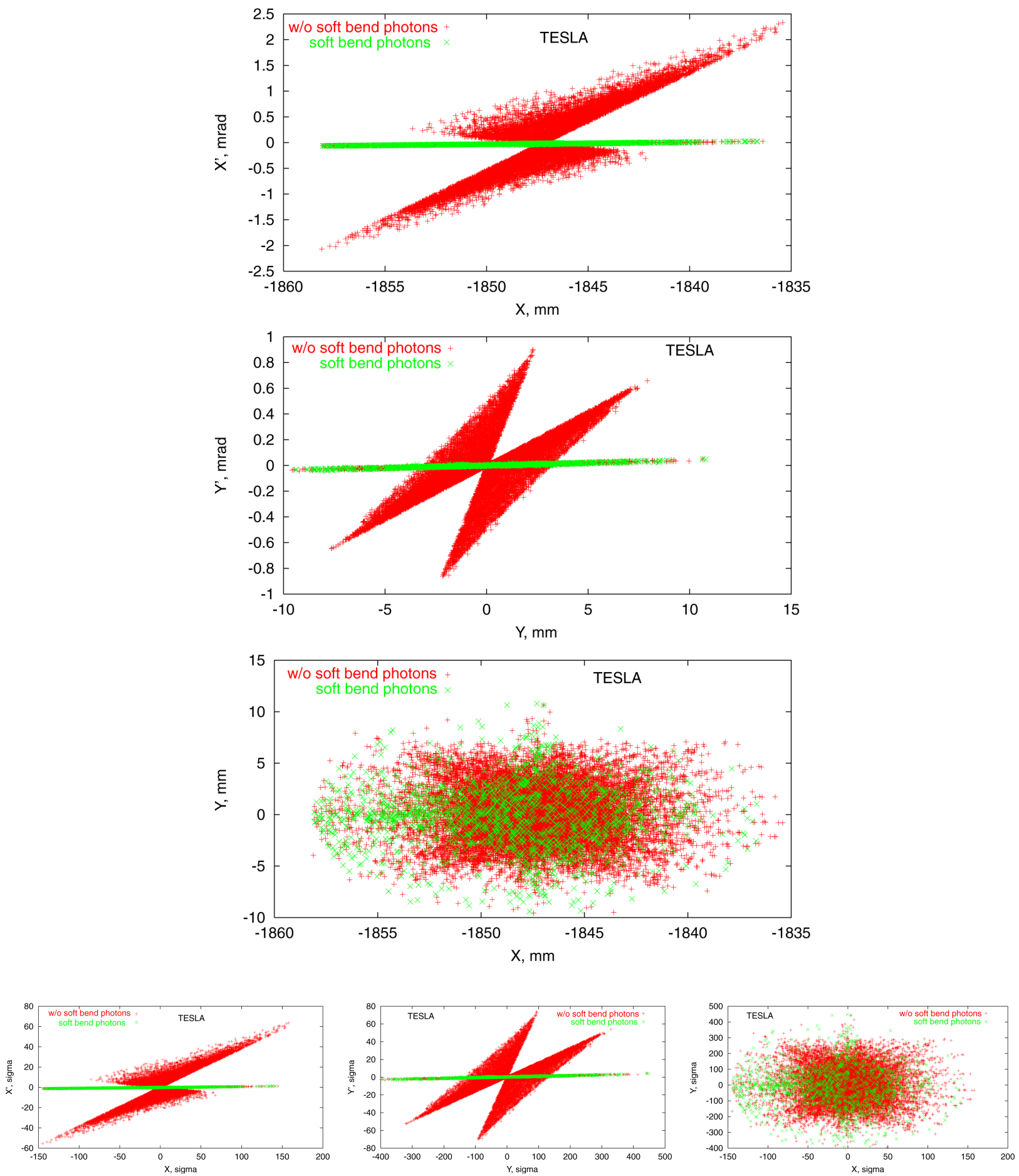

Figure 38: TESLA synchrotron radiation population from beam halo in the phase plane at the . detector mask downstream from the IP. Green (grey) are photons from the soft bend. The bottom pictures are the same plots in units of beam sigma. 

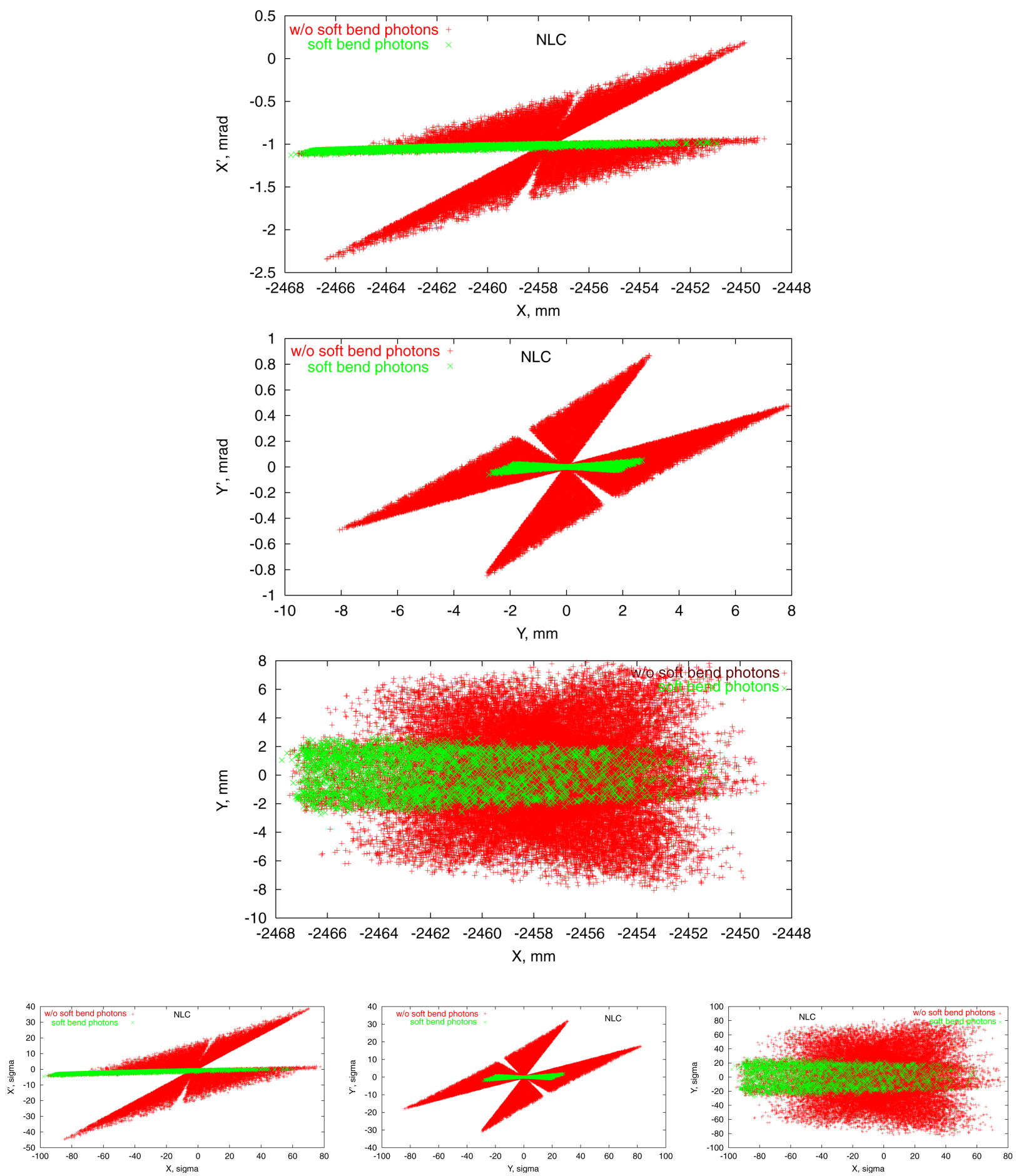

Figure 39: NLC synchrotron radiation population from beam halo in the phase plane at the . luminosity monitor downstream from the IP. Green (grey) are photons from the soft bend. The bottom pictures are the same plots in units of beam sigma. 

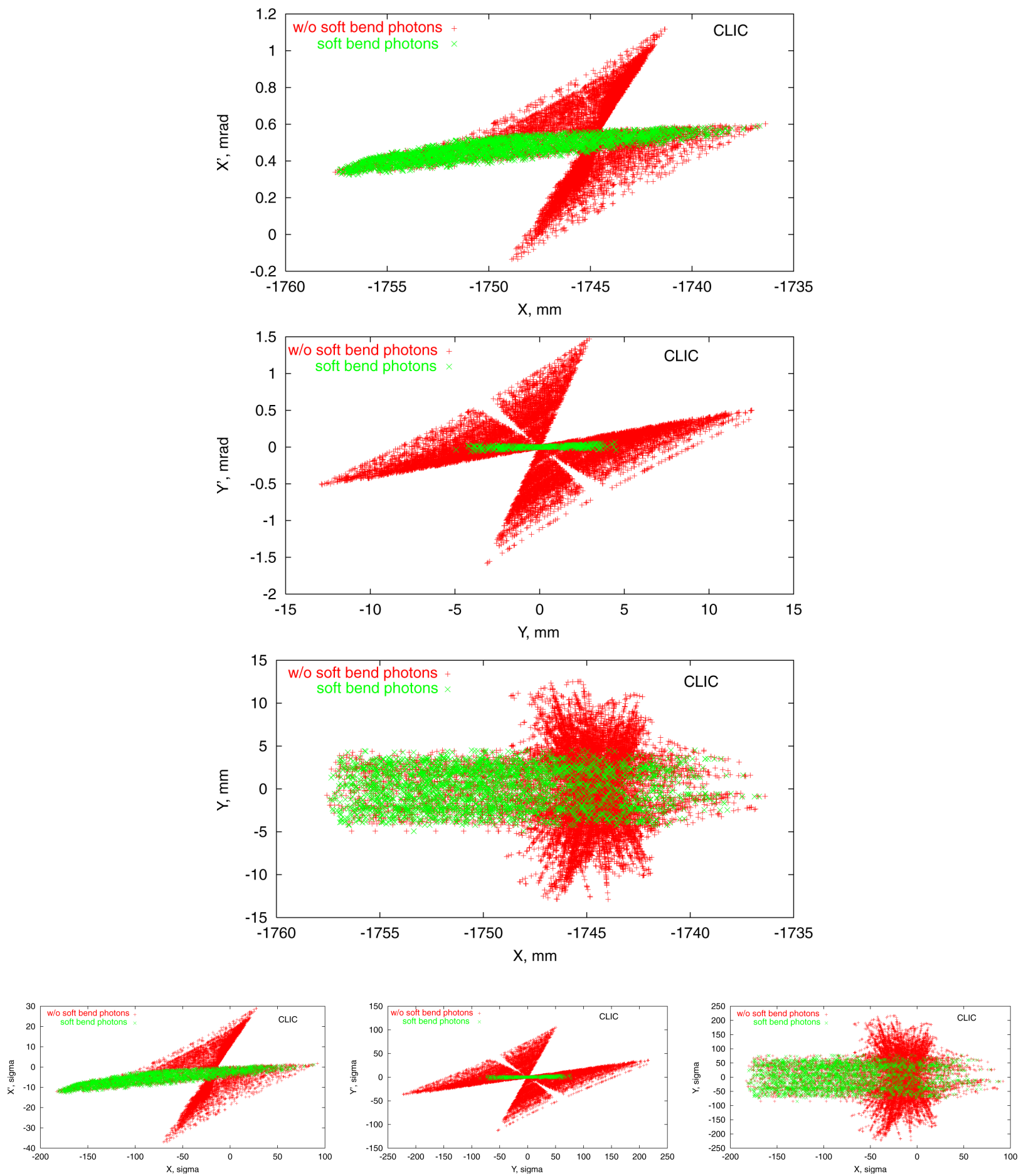

Figure 40: CLIC synchrotron radiation population from beam halo in the phase plane at the . detector mask downstream from the IP. Green (grey) are photons from the soft bend. The bottom pictures are the same plots in units of beam sigma. 